\title{
On dilation symmetries arising from scaling limits
}

\author{
Henning Bostelmann* Claudio D’Antoni*† Gerardo Morsella*†‡ \\ Università di Roma "Tor Vergata", Dipartimento di Matematica, \\ Via della Ricerca Scientifica, 00133 Roma, Italy \\ E-mail addresses: (bostelma, dantoni, morsella)@mat .uniroma2.it
}

December 27, 2008

\begin{abstract}
Quantum field theories, at short scales, can be approximated by a scaling limit theory. In this approximation, an additional symmetry is gained, namely dilation covariance. To understand the structure of this dilation symmetry, we investigate it in a nonperturbative, model independent context. To that end, it turns out to be necessary to consider nonpure vacuum states in the limit. These can be decomposed into an integral of pure states; we investigate how the symmetries and observables of the theory behave under this decomposition. In particular, we consider several natural conditions of increasing strength that yield restrictions on the decomposed dilation symmetry.
\end{abstract}

\section{Introduction}

In the analysis of quantum field theories, the notion of scaling limits plays an important role. The physical picture underlying this mathematical concept is as follows: One considers measurements in smaller and smaller space-time regions, at the same time increasing the energy content of the states involved, so that the characteristic action scale remains constant. Passing to the limit of infinitesimal scales, one obtains a new quantum field theory, the scaling limit of the original model. The scaling limit theory can be seen as an approximation of the full theory in the short-distance regime. However, it may differ significantly from the full theory in fundamental aspects, for example regarding its charge structure: In quantum chromodynamics, it is expected that confined charges (color) appear in the limit theory, but are not visible as such in the full theory.

The virtues of the scaling limit theory include that it is typically simpler than the original one. In fact, in relevant examples, one expects it to be interactionless (asymptotic freedom). But even where this is not the case, the limit theory should possess an additional symmetry: It should be dilation covariant, since any finite masses in the original model can be neglected in the limit of large energies.

On the mathematical side, a very natural description of scaling limits has been given by Buchholz and Verch [BV95]. This description, formulated in the $\mathrm{C}^{*}$ algebraic framework

\footnotetext{
*Supported in parts by the EU network "Noncommutative Geometry" (MRTN-CT-2006-0031962)

${ }^{\dagger}$ Supported in parts by PRIN-MIUR and GNAMPA-INDAM

${ }^{\ddagger}$ Supported in parts by the Scuola Normale Superiore, Pisa, Italy
} 
of local quantum physics [Haa96], originates directly from the physical notions, and avoids any additional input motivated merely on the technical side, such as a rescaling of coupling constants or mass parameters, or the choice of renormalization factors for quantum fields. This has the advantage of allowing an intrinsic, model-independent description of the short distance properties of the theory at hand. In particular, it has been successfully applied to the analysis of the charge structure of the theory in the scaling limit and to the intrinsic characterization of charge confinement Buc96b, DMV04]. While the framework of Buchholz and Verch seems rather abstract at first, it has recently been shown that it reproduces the usual picture of multiplicative field renormalization in typical cases [BDM09].

The approach of [BV95] is based on the notion of the scaling algebra $\underline{\mathfrak{A}}$, which consists roughly speaking - of sequences of observables $\lambda \mapsto \underline{A}_{\lambda}$ at varying scale $\lambda$, uniformly bounded in norm, and subject to certain continuity conditions. (We shall recall the precise definition in Sec. 2.1.) The task of passing to the scaling limit is then reduced to finding a suitable state $\underline{\omega}_{0}$ on the $\mathrm{C}^{*}$ algebra $\underline{\mathfrak{A}}$ that represents the vacuum of the limit theory; it is constructed as a limit of vacuum states at finite scales. The limit theory itself is then obtained by a standard GNS construction with respect to $\underline{\omega}_{0}$.

It should be easy in this context to describe the additional dilation symmetry that arises in the scaling limit. In fact, the scaling algebra $\mathfrak{A}$ carries a very natural representation $\mu \mapsto \underline{\delta}_{\mu}$ of the dilation group, which acts by shifting the argument of the functions $\lambda \mapsto \underline{A}_{\lambda}$ : $\underline{\delta}_{\mu}(\underline{A})_{\lambda}=\underline{A}_{\mu \lambda}$. However, things turn out to be more involved: The limit states $\underline{\omega}_{0}$ described in BV95 are not invariant under this group action, and thus one does not obtain a canonical group representation in the limit Hilbert space. In BDM09], generalized limit states have been introduced, some of which are invariant under dilations, and give rise to a unitary implementation of the dilation group in the limit theory. However, these dilation invariant limit states are never pure; rather they arise as a mixture of states of the Buchholz-Verch type, which are pure in $2+1$ or more space-time dimensions.

The object of the present paper is to analyze this generalized class of limit states in more detail, in order to describe the structure of the dilation symmetry associated with the dilation invariant ones. In particular we will show that, as briefly mentioned in [BDM09], the decomposition of these states in pure (Buchholz-Verch type) states gives rise to a direct integral decomposition of the limit Hilbert space, which also induces a decomposition of observables and of Poincaré symmetries. It should be noted here that the entire construction is complicated by the fact that uncountably many extremal states are involved in this decomposition, and that the measure space underlying the direct integral is of a very general nature. Because of this, we need to use a notion of direct integral of Hilbert spaces which is more general than the one previously employed in the quantum field theory literature [DS85].

It is also of interest to discuss how the special but physically important class of theories with a unique scaling limit, as defined in [BV95], fits into our generalized framework. It turns out that, up to some technical conditions, uniqueness of the scaling limit in the BuchholzVerch framework is equivalent to the factorization of our generalized scaling limit into a tensor product of an irreducible scaling limit theory and a commutative part, which is just the image under the scaling limit representation of the center of the scaling algebra. In particular we show that such factorization holds for a restricted class of theories, those with a convergent scaling limit. This class includes in particular dilation invariant theories and free field models. The technical conditions referred to above consist in a suitable separability requirement of the scaling limit Hilbert space, which is needed in order to be able to employ the full power of direct integrals theory. As a matter of fact, such separability condition is a consequence of 
a refined version of the Haag-Swieca compactness condition.

With these results at hand, it is possible to discuss the structure of the unitarily implemented dilation symmetry in dilation invariant scaling limit states. The outcome is that in general the dilations do not decompose, not even in the factorizing situation. Rather, the dilations intertwine in a suitable sense the different pure limit states that occur in the direct integral decomposition. A complete factorization of the dilation symmetry is however obtained in the convergent scaling limit case. For such theories, therefore, one gets a unitary implementation of the dilation symmetry in the pure limit theory.

The remainder of this paper is organized as follows: First, in Sec. 2, we recall the notion of scaling limits in the algebraic approach to quantum field theory, and generalize some fundamental results of [BV95] to our situation. In Sec. 3, we establish the direct integral decomposition mentioned above, including a decomposition of local observables and Poincaré symmetries. Sec. 4 contains a discussion of unique scaling limits as a special case. We define several conditions that generalize the notion from [BV95], and discuss relations between them. Then, in Sec. 5, we analyze the structure of dilation symmetries in the limit Hilbert space, and their decomposition along the direct integral, on different levels of generality. In Sec. 6 we propose a stronger version of the Haag-Swieca compactness condition and we show that it implies the separability property used in the analysis of Sec. 4. Sec. 7 discusses some simple models as examples, showing in particular that these fulfill all of our conditions proposed in Sec. 4 and 6. We conclude with a brief outlook in Sec. 8, The appendix reviews the concept of direct integrals of Hilbert spaces, which we need in a more general variant than covered in the standard literature.

\section{Definitions and general results}

We shall first recall the definition of scaling limits in the algebraic approach to quantum field theory, and prove some fundamental results regarding uniqueness of the limit vacuum state and regarding geometric modular action.

\subsection{The setting}

We consider quantum field theory on $(s+1)$ dimensional Minkowski space. For our analysis, we work entirely within the framework of algebraic quantum field theory [Haa96], where observables localized in a space-time region $\mathcal{O}$ are described by the selfadjoint elements of a $\mathrm{C}^{*}$ algebra $\mathfrak{A}(\mathcal{O})$. Let us repeat the formal definition of a quantum field theoretical model in this context.

Definition 2.1. Let $\mathcal{G}$ be a Lie group of point transformations of Minkowski space that includes the translation group. A local net of algebras with symmetry group $\mathcal{G}$ is a net of algebras $\mathfrak{A}$ together with a representation $g \mapsto \alpha_{g}$ of $\mathcal{G}$ as automorphisms of $\mathfrak{A}$, such that

(i) $\left[A_{1}, A_{2}\right]=0$ if $\mathcal{O}_{1}, \mathcal{O}_{2}$ are two spacelike separated regions, and $A_{i} \in \mathfrak{A}\left(\mathcal{O}_{i}\right)$;

(ii) $\alpha_{g} \mathfrak{A}(\mathcal{O})=\mathfrak{A}(g \cdot \mathcal{O})$ for all $\mathcal{O}, g$.

We call $\mathfrak{A}$ a net in a positive energy representation if, in addition, the $\mathfrak{A}(\mathcal{O})$ are $W^{*}$ algebras acting on a common Hilbert space $\mathcal{H}$, and 
(iii) there is a strongly continuous unitary representation $g \mapsto U(g)$ of $\mathcal{G}$ on $\mathcal{H}$ such that $\alpha_{g}=\operatorname{ad} U(g)$;

(iv) the joint spectrum of the generators of translations $U(x)$ lies in the closed forward light cone $\overline{\mathcal{V}}_{+}$;

(v) there exists a vector $\Omega \in \mathcal{H}$ which is invariant under all $U(g)$ and cyclic for $\mathfrak{A}$.

$W e$ call $\mathfrak{A}$ a net in the vacuum sector if, in addition,

(vi) the vector $\Omega$ is unique (up to scalar factors) as an invariant vector for the translation group.

Our approach is to start from a local net $\mathfrak{A}$ in the vacuum sector, with the Poincaré group $\mathcal{P}_{+}^{\uparrow}$ as its symmetry group; this net $\mathfrak{A}$ will be kept fixed in all that follows. Our aim is to describe the short-distance scaling limit of $\mathfrak{A}$. Following BV95, we define $\underline{\mathfrak{B}}$ to be the set of bounded functions $\underline{B}: \mathbb{R}_{+} \rightarrow \mathcal{B}(\mathcal{H}), \lambda \mapsto \underline{B}_{\lambda}$. Equipped with pointwise addition, multiplication, and $*$ operation, and with the norm $\|\underline{B}\|=\sup _{\lambda}\left\|\underline{B}_{\lambda}\right\|$, the set $\underline{\mathfrak{B}}$ becomes a $\mathrm{C}^{*}$ algebra. Let $\underline{\mathcal{G}}$ be the group formed by Poincaré transformations and dilations; we will write $\underline{\mathcal{G}} \ni g=(\mu, x, \Lambda)$ with $\mu \in \mathbb{R}_{+}, x \in \mathbb{R}^{s+1}$, and $\Lambda$ a Lorentz matrix. $\underline{\mathcal{G}}$ acts on $\underline{\mathfrak{B}}$ via a representation $\underline{\alpha}$, given by

$$
\left(\underline{\alpha}_{g} \underline{B}\right)_{\lambda}=\alpha_{\lambda \mu x, \Lambda}\left(\underline{B}_{\lambda \mu}\right) \quad \text { for } g=(\mu, x, \Lambda) \in \underline{\mathcal{G}}, \underline{B} \in \underline{\mathfrak{B}},
$$

where $\alpha$ is the Poincaré group representation on $\mathfrak{A}$. Note the rescaling of translations with the scale parameter $\lambda$. We now define new local algebras as subsets of $\underline{\mathfrak{B}}$ :

$$
\underline{\mathfrak{A}}(\mathcal{O}):=\left\{\underline{A} \in \underline{\mathfrak{B}} \mid \underline{A}_{\lambda} \in \mathfrak{A}(\lambda \mathcal{O}) \text { for all } \lambda>0 ; g \mapsto \underline{\alpha}_{g}(\underline{A}) \text { is norm continuous }\right\} .
$$

This is a net of local algebras in the sense of Def. 2.1, with the enlarged symmetry group $\underline{\mathcal{G}}$ BDM09. We denote by $\underline{\mathfrak{A}}$ the associated quasilocal algebra, i.e. the inductive limit of $\underline{\mathfrak{A}}(\overline{\mathcal{O}})$ as $\mathcal{O} \nearrow \mathbb{R}^{s+1}$. This $\underline{\mathfrak{A}}$ is called the scaling algebra. Note that $\underline{\mathfrak{A}}$ has a large center $\mathfrak{Z}(\underline{\mathfrak{A}})$, consisting of all operators $\underline{A}$ of the form $\underline{A}_{\lambda}=f(\lambda) \mathbf{1}$, where $f: \mathbb{R}_{+} \rightarrow \mathbb{C}$ is a uniformly continuous function on $\mathbb{R}_{+}$as a group under multiplication. We often identify $\underline{A} \in \mathfrak{Z}(\underline{\mathfrak{A}})$ with the function $f$ without further notice.

For a description of the scaling limit, we first consider states on $\mathfrak{Z}(\underline{\mathfrak{A}})$. Let $\mathrm{m}$ be a mean on the uniformly continuous functions 11 on $\mathbb{R}_{+}$, i.e., a positive normalized linear functional on the commutative $\mathrm{C}^{*}$ algebra $\mathfrak{Z}(\underline{\mathfrak{A}})$. We say that $\mathrm{m}$ is asymptotic if $\mathrm{m}(f)=\lim _{\lambda \rightarrow 0} f(\lambda)$ whenever the limit on the right-hand side exists; or, equivalently, if $\mathrm{m}(f)=0$ whenever $f(\lambda)=0$ for small $\lambda$. Asymptotic means are, in this sense, generalizations of the limit $\lambda \rightarrow 0$. Further we consider two important classes of means:

(i) $\mathrm{m}$ is called multiplicative if $\mathrm{m}(f g)=\mathrm{m}(f) \mathrm{m}(g)$ for all functions $f, g$.

(ii) $\mathrm{m}$ is called invariant if $\mathrm{m}\left(f_{\mu}\right)=\mathrm{m}(f)$ for all functions $f$ and all $\mu>0$, where $f_{\mu}=f(\mu \cdot)$.

\footnotetext{
${ }^{1}$ In contrast to [BDM09, we do not consider means on the bounded functions on $\mathbb{R}_{+}$, but rather on the uniformly continuous functions. While all of them can be extended to the bounded functions, these extensions do not play a role in our current investigation.
} 
It is an important fact that (i) and (ii) are mutually exclusive; there are no multiplicative invariant means in our situation (cf. [Mit66]).

We now extend these "generalized limits" of functions to a limit of operator sequences, using a projection technique. Let $\omega=(\Omega|\cdot| \Omega)$ be the vacuum state of $\mathfrak{A}$. This state induces a projector (or conditional expectation) in $\underline{\mathfrak{A}}$ onto $\mathfrak{Z}(\underline{\mathfrak{A}})$, which we denote by the same symbol:

$$
\omega: \underline{\mathfrak{A}} \rightarrow \mathfrak{Z}(\underline{\mathfrak{A}}), \quad(\omega(\underline{A}))_{\lambda}=\omega\left(\underline{A}_{\lambda}\right) \mathbf{1} .
$$

Using this projector, any mean $\mathrm{m}$ defines a state $\underline{\omega}_{\mathrm{m}}$ on $\underline{A}$ by $\underline{\omega}_{\mathrm{m}}:=\mathrm{m} \circ \omega$. If here $\mathrm{m}$ is asymptotic, we call $\underline{\omega}_{m}$ a limit state, and typically denote it by $\underline{\omega}_{0}$. These are the states that correspond to scaling limits of the quantum field theory. Since there is a one-to-one correspondence between asymptotic means and limit states, we will usually work with the state $\underline{\omega}_{0}$ only, and not refer to the mean m explicitly. A limit state $\underline{\omega}_{0}$ will be called multiplicative 2 or invariant if the corresponding mean has this property. Multiplicative limit states correspond to those considered by Buchholz and Verch in BV95. Every other limit state arises from these by convex combinations and weak* limits; this follows directly from the property of states on the commutative algebra $\mathfrak{Z}(\underline{\mathfrak{A}})$.

Given a limit state $\underline{\omega}_{0}$, we can obtain the limit theory via a GNS construction: Let $\pi_{0}$ be the GNS representation of $\underline{\mathfrak{A}}$ with respect to $\underline{\omega}_{0}$, and $\mathcal{H}_{0}$ the representation space, with GNS vector $\Omega_{0}$. Denoting by $\mathcal{G}_{0}$ the subgroup of $\underline{\mathcal{G}}$ under which $\underline{\omega}_{0}$ is invariant, we canonically obtain a strongly continuous unitary representation of $\mathcal{G}_{0}$ on $\mathcal{H}_{0}$ by setting $U_{0}(g) \pi_{0}(\underline{A}) \Omega_{0}:=$ $\pi_{0}\left(\underline{\alpha}_{g}(\underline{A})\right) \Omega_{0}, g \in \mathcal{G}_{0}$. The subgroup $\mathcal{G}_{0}$ contains the Poincaré group; and if $\underline{\omega}_{0}$ is invariant, then $\mathcal{G}_{0}=\underline{\mathcal{G}}$. The translation part of $U_{0}$ fulfills the spectrum condition [BDM09]. Setting $\mathfrak{A}_{0}(\mathcal{O}):=\pi_{0}(\underline{\mathfrak{A}}(\mathcal{O}))^{\prime \prime}$, one obtains a local net $\mathfrak{A}_{0}$ with symmetry group $\mathcal{G}_{0}$ in a positive energy representation: the limit theory.

\subsection{Multiplicity of the vacuum state}

If $\underline{\omega}_{0}$ is a multiplicative limit state, its restriction to $\mathfrak{Z}(\underline{\mathfrak{A}})$ is pure. It has been shown in BV95] that in the case $s \geq 2$, this property extends to the entire theory: $\underline{\omega}_{0}$ is a pure vacuum state on $\underline{\mathfrak{A}}$, and $\pi_{0}$ is an irreducible representation. On the other hand, if $\underline{\omega}_{0}$ is not multiplicative, the same must be false, since already $\pi_{0}\lceil\mathfrak{Z}(\underline{\mathfrak{A}})$ is reducible. However, we shall show that this property of the center is the only "source" of reducibility: namely one has $\pi_{0}(\underline{\mathfrak{A}})^{\prime}=\pi_{0}(\mathfrak{Z}(\underline{\mathfrak{A}}))^{\prime}$.

We need some preparations to prove this. In the following, set $\mathfrak{Z}_{0}:=\pi_{0}(\mathfrak{Z}(\underline{\mathfrak{A}}))^{\prime \prime}$, and let $\mathcal{H}_{\mathfrak{Z}}:=\operatorname{clos}\left(\mathfrak{Z}_{0} \Omega_{0}\right) \subset \mathcal{H}_{0}$ be the representation space of the commutative algebra.

Lemma 2.2. Let $P_{\mathfrak{Z}} \in \mathcal{B}\left(\mathcal{H}_{0}\right)$ be the orthogonal projector onto $\mathcal{H}_{\mathfrak{Z}}$. If $s \geq 2$, then $P_{\mathfrak{Z}} \in$ $\pi_{0}(\underline{\mathfrak{A}})^{\prime \prime}$, and $\mathcal{H}_{\mathfrak{Z}}$ is the space of all translation-invariant vectors in $\mathcal{H}_{0}$.

Proof. As a consequence of the spectrum condition in the theory $\mathfrak{A}_{0}$, it is known Ara64 that the translation operators $U_{0}(x)$ are contained in $\pi_{0}(\underline{\mathfrak{A}})^{\prime \prime}$. Now let $U_{\infty}$ be an ultraweak cluster point of $U_{0}(x)$ as $x$ goes to spacelike infinity on some fixed sequence within the time-0 plane. (Such cluster points exist by the Alaoglu-Bourbaki theorem.) Then $U_{\infty} \in \pi_{0}(\underline{\mathfrak{A}})^{\prime \prime}$; we will show $U_{\infty}=P_{\mathfrak{Z}}$.

To that end, we first note that

$$
\underline{\omega}_{0}(\underline{A} \underline{B})=\underline{\omega}_{0}(\omega(\underline{A}) \underline{B}) \quad \text { for all } \underline{A} \in \underline{\mathfrak{A}}, \underline{B} \in \mathfrak{Z}(\underline{\mathfrak{A}}),
$$

\footnotetext{
${ }^{2}$ For clarity, we note that a multiplicative limit state, by this definition, is not a multiplicative functional on $\underline{\mathfrak{A}}$, but is multiplicative only on the center $\mathfrak{Z}(\underline{\mathfrak{A}})$.
} 
which follows directly from the definition of $\underline{\omega}_{0}$. Now we make use of the cluster property of the vacuum at finite scales. As in [BV95, Lemma 4.3], one can obtain the following norm estimate in the algebra $\mathfrak{A}$ :

$$
\left\|\omega\left(\underline{A} \underline{\alpha}_{x} \underline{B}\right)-\omega(\underline{A}) \omega(\underline{B})\right\| \leq c \frac{r^{s}}{|x|^{s-1}}(\|\underline{A}\|\|\underline{\dot{B}}\|+\|\underline{\dot{A}}\|\|\underline{B}\|)
$$

for fixed $r>0, x$ in the time- 0 plane with $|x|>3 r$, and for $\underline{A}, \underline{B}$ chosen from some normdense subset of $\mathfrak{A}\left(\mathcal{O}_{r}\right)$, with $\mathcal{O}_{r}$ being the standard double cone of radius $r$ around the origin. Here $c>0$ is some constant, and the dot denotes the time derivative. This implies that as $|x| \rightarrow \infty$,

$$
\lim _{x} \underline{\omega}_{0}\left(\underline{A}_{\alpha_{x}} \underline{B}\right)=\underline{\omega}_{0}(\omega(\underline{A}) \omega(\underline{B}))
$$

for these $\underline{A}, \underline{B}$. Now it follows from Eq. (2.4) - with $\omega(\underline{B})$ in place of $\underline{B}$ - that

$$
\left(\pi_{0}(\underline{A}) \Omega_{0} \mid U_{\infty} \pi_{0}(\underline{B}) \Omega_{0}\right)=\lim _{x} \underline{\omega}_{0}\left(\underline{A} \underline{\alpha}_{x} \underline{B}\right)=\left(\pi_{0}(\underline{A}) \Omega_{0} \mid \pi_{0}(\omega(\underline{B})) \Omega_{0}\right) .
$$

Continuing this relation from the dense sets chosen, this means

$$
U_{\infty} \pi_{0}(\underline{B}) \Omega_{0}=\pi_{0}(\omega(\underline{B})) \Omega_{0} \quad \text { for all } \underline{B} \in \underline{\mathfrak{A}} .
$$

This shows that $U_{\infty}^{2}=U_{\infty}$, and $\operatorname{img} U_{\infty}=\mathcal{H}_{\mathfrak{Z}}$. Also, again applying Eq. (2.4), one obtains $U_{\infty}^{*}=U_{\infty}$. Thus $U_{\infty}$ is the unique orthogonal projector onto $\mathcal{H}_{\mathfrak{Z}}$.-For the last part, note that translations act trivially on $\mathcal{H}_{\mathfrak{Z}}$, and that $U_{\infty}$ leaves all translation-invariant vectors unchanged; so $\mathcal{H}_{\mathfrak{Z}}$ is the space of all translation-invariant vectors.

We are now ready to prove the announced result about the commutant of $\pi_{0}(\underline{\mathfrak{A}})$.

Theorem 2.3. Let $s \geq 2$. Let $\underline{\omega}_{0}$ be a limit state, and let $\pi_{0}$ be the corresponding GNS representation. Then $\pi_{0}(\underline{\mathfrak{A}})^{\prime}=\pi_{0}(\mathfrak{Z}(\underline{\mathfrak{A}}))^{\prime \prime}$.

Proof. Let $B \in \pi_{0}(\underline{\mathfrak{A}})^{\prime}$. By Lemma $2.2, B$ commutes with $P_{\mathfrak{Z}}$; hence $B \mathcal{H}_{\mathfrak{Z}} \subset \mathcal{H}_{\mathfrak{Z}}$, and $B\left\lceil\mathcal{H}_{\mathfrak{Z}} \in \mathfrak{B}\left(\mathcal{H}_{\mathfrak{Z}}\right)\right.$ is well-defined. As $\mathfrak{Z}_{0} \subset \pi_{0}(\underline{\mathfrak{A}})^{\prime \prime}$, we know that

$$
\left[B \left\lceil\mathcal{H}_{\mathfrak{Z}}, C\left\lceil\mathcal{H}_{\mathfrak{Z}}\right]=0 \quad \text { for all } C \in \mathfrak{Z}_{0}\right.\right.
$$

as an equation in $\mathfrak{B}\left(\mathcal{H}_{\mathfrak{Z}}\right)$. Since $\mathfrak{Z}_{0}\left\lceil\mathcal{H}_{\mathfrak{Z}}\right.$ is a maximal abelian algebra in $\mathfrak{B}\left(\mathcal{H}_{\mathfrak{Z}}\right)$ [BR79, Lemma 4.3.15], there exists $C \in \mathfrak{Z}_{0}$ with $B\left\lceil\mathcal{H}_{\mathfrak{Z}}=C\left\lceil\mathcal{H}_{\mathfrak{Z}}\right.\right.$. Now for any $A \in \pi_{0}(\underline{\mathfrak{A}})^{\prime \prime}$, we can compute

$$
B A \Omega_{0}=A B \Omega_{0}=A C \Omega_{0}=C A \Omega_{0} .
$$

Since $\Omega_{0}$ is cyclic for $\pi_{0}$, this implies $B=C$. Thus $\pi_{0}(\underline{\mathfrak{A}})^{\prime} \subset \mathfrak{Z}_{0}$. The reverse inclusion is trivial.

It should be noted that the same theorem does not hold in $1+1$ space-time dimensions. In this case, it is known even in free field theory [BV98, Sec. 4] that the algebra $\pi_{0}(\underline{\mathfrak{A}})$ has a large center, even if $\pi_{0}(\mathfrak{Z}(\underline{\mathfrak{A}}))=\mathbb{C} \mathbf{1}$.

We can now easily reproduce the known results for multiplicative limit states. In this case, the GNS representation of the abelian algebra $\mathfrak{Z}(\underline{\mathfrak{A}})$ for the state $\underline{\omega}_{0}$ must be irreducible; thus $\mathfrak{Z}_{0}=\mathbb{C} \mathbf{1}$, and $\operatorname{dim} \mathcal{H}_{\mathfrak{Z}}=1$. The above results imply:

Corollary 2.4. Let $\underline{\omega}_{0}$ be a multiplicative limit state, and let $s \geq 2$. Then $\Omega_{0}$ is unique up to a scalar factor as an invariant vector for the translations $U_{0}(x)$, and the representation $\pi_{0}$ is irreducible. $\mathfrak{A}_{0}$ is a net in the vacuum sector in the sense of Def. 2.1. 


\subsection{Wedge algebras and geometric modular action}

While we have defined the scaling limit in terms of local algebras for bounded regions, it is also worthwhile to consider algebras associated with unbounded, in particular wedge-shaped regions. This is particularly important in the context of charge analysis for the limit theory DMV04, DM06]. While we do not enter this topic here, and do not build on it in the following, we wish to discuss briefly how wedge algebras and the condition of geometric modular action fit into our context. Again, this transfers results of BV95 to our generalized class of limit states.

Let $\mathcal{W}$ be a wedge region, i.e. $\mathcal{W}$ is a Poincaré transform of the right wedge

$$
\mathcal{W}_{+}=-\mathcal{W}_{-}=\left\{x \in \mathbb{R}^{4} \mid x \cdot e_{+}<0\right\}, \quad \text { where } e_{ \pm}:=( \pm 1,1,0,0) .
$$

Note that $\left(\overline{\mathcal{W}}_{+}\right)^{\prime}=\mathcal{W}_{-}$. We introduce the one-parameter group $\left(\Lambda_{t}\right)_{t \in \mathbb{R}}$ of Lorentz boosts leaving $\mathcal{W}_{+}$invariant, fixed by $\Lambda_{t} e_{ \pm}=\exp ( \pm t) e_{ \pm}$, and acting as the identity on the edge $\left(e_{ \pm}\right)^{\perp}$ of $\mathcal{W}_{+}$. Let furthermore $j$ be the inversion with respect to the edge of $\mathcal{W}_{+}$, i.e. $j e_{ \pm}=-e_{ \pm}$ and $j=\mathbf{1}$ on $\left(e_{ \pm}\right)^{\perp}$. Note that $j^{2}=\mathbf{1}$ and $j \mathcal{W}_{+}=\mathcal{W}_{-}$.

For a local net of algebras (resp. for a net of algebras in a positive energy representation) $\mathcal{O} \mapsto \mathfrak{A}(\mathcal{O})$, we define the algebra $\mathfrak{A}(\mathcal{W})$ associated to the wedge $\mathcal{W}$ as the $\mathrm{C}^{*}$-algebra (resp. $\mathrm{W}^{*}$-algebra) generated by the algebras $\mathfrak{A}(\mathcal{O})$, where $\mathcal{O}$ is any double cone whose closure is contained in $\mathcal{W}(\mathcal{O} \subset \subset \mathcal{W}$ in symbols). With these definitions, we can adapt the arguments in [BV95, Lemma 6.1], which do not depend on irreducibility of the net. It is then straightforward to verify that, for a net $\mathfrak{A}$ in a positive energy representation, the vacuum vector $\Omega$ is cyclic and separating for all wedge algebras $\mathfrak{A}(\mathcal{W})$. This allows us to introduce the notion of geometric modular action.

Definition 2.5. Let $\mathfrak{A}$ be a local net in a positive energy representation, and denote by $\Delta$, $J$ the modular objects associated to $\mathfrak{A}\left(\mathcal{W}_{+}\right), \Omega$. The net $\mathfrak{A}$ is said to satisfy the condition of geometric modular action if there holds

$$
\begin{array}{rlrl}
\Delta^{i t} & =U\left(\Lambda_{2 \pi t}\right), & t \in \mathbb{R}, \\
J U(x, \Lambda) J & =U(j x, j \Lambda j), & (x, \Lambda) \in \mathcal{P}_{+}^{\uparrow}, \\
J \mathfrak{A}(\mathcal{O}) J & =\mathfrak{A}(j \mathcal{O}) . & &
\end{array}
$$

If $\mathfrak{A}$ satisfies the condition of geometry modular action, then it also satisfies wedge duality, since, according to Tomita-Takesaki theory and equation (函),

$$
\mathfrak{A}\left(\mathcal{W}_{+}\right)^{\prime}=J \mathfrak{A}\left(\mathcal{W}_{+}\right) J=\mathfrak{A}\left(\mathcal{W}_{-}\right) .
$$

This also implies that $\mathfrak{A}$ satisfies essential Haag duality, i.e. that the dual net $\mathfrak{A}^{d}$ of $\mathfrak{A}$, defined on double cones $\mathcal{O}$ as

$$
\mathfrak{A}^{d}(\mathcal{O}):=\bigwedge_{\mathcal{W} \supset \mathcal{O}} \mathfrak{A}(\mathcal{W})
$$

is local and such that $\mathfrak{A}(\mathcal{O}) \subset \mathfrak{A}^{d}(\mathcal{O})$ for each double cone $\mathcal{O}$.

From now on, let $\mathfrak{A}$ be a net in the vacuum sector, and $\underline{\omega}_{0}$ a scaling limit state, with $\pi_{0}$ the corresponding scaling limit representation. It holds that $\pi_{0}(\underline{\mathfrak{A}}(\mathcal{W}))^{\prime \prime}=\mathfrak{A}_{0}(\mathcal{W})$, since clearly

$$
\pi_{0}(\underline{\mathfrak{A}}(\mathcal{W}))=\overline{\bigcup_{\mathcal{O} \subset \subset \mathcal{W}} \pi_{0}(\underline{\mathfrak{A}}(\mathcal{O}))}
$$


and therefore

$$
\pi_{0}(\underline{\mathfrak{A}}(\mathcal{W}))^{\prime}=\bigwedge_{\mathcal{O} \subset \subset \mathcal{W}} \pi_{0}(\underline{\mathfrak{A}}(\mathcal{O}))^{\prime}=\bigwedge_{\mathcal{O} \subset \subset \mathcal{W}} \mathfrak{A}_{0}(\mathcal{O})^{\prime}=\mathfrak{A}_{0}(\mathcal{W})^{\prime} .
$$

Proposition 2.6. Assume that $\mathfrak{A}$ satisfies the condition of geometric modular action. Then for each limit state $\underline{\omega}_{0}$, the corresponding limit theory $\mathfrak{A}_{0}$ also satisfies the condition of geometric modular action.

Proof. It's a straightforward adaptation of the proofs of Lemma 6.2 and Proposition 6.3 of [BV95]. The only point which is worth mentioning is the proof that $\underline{\omega}_{0}$ is a KMS state (at inverse temperature $2 \pi)$ for the algebra $\underline{\mathfrak{A}}\left(\mathcal{W}_{+}\right)$with respect to the one-parameter group of automorphisms $\left(\underline{\alpha}_{\Lambda_{t}}\right)_{t \in \mathbb{R}}$, which goes as follows. Let $\mathrm{m}$ be the mean which induces $\underline{\omega}_{0}$. Then $\mathrm{m}$ is a weak* limit of convex combinations of multiplicative means, and therefore $\underline{\omega}_{0}$ is a weak* limit of convex combinations of multiplicative limit states. For such states, the arguments in [BV95, Lemma 6.2] show that they are KMS on $\underline{A}\left(\mathcal{W}_{+}\right)$, and therefore, the set of KMS states at a fixed inverse temperature being convex and weak* closed [BR81, Thm. 5.3.30], this holds also for $\underline{\omega}_{0}$.

\section{Decomposition theory}

Our aim is now to decompose an arbitrary limit state $\underline{\omega}_{0}$ into "simple" limit states of the Buchholz-Verch type, and to obtain corresponding decompositions of the relevant objects in the limit theory. We start by proving an integral decomposition which is a consequence of standard results.

Proposition 3.1. Let $\underline{\omega}_{0}$ be a limit state. There exists a compact Hausdorff space $\mathcal{Z}$, a regular Borel probability measure $\nu$ on $\mathcal{Z}$, and for each $z \in \mathcal{Z}$ a multiplicative limit state $\underline{\omega}_{z}$, such that

$$
\underline{\omega}_{0}(\underline{A})=\int_{\mathcal{Z}} d \nu(z) \underline{\omega}_{z}(\underline{A}) \quad \text { for all } \underline{A} \in \underline{\mathfrak{A}} .
$$

Further, the map $\mathfrak{Z}(\underline{\mathfrak{A}}) \rightarrow \mathcal{C}(\mathcal{Z}), \underline{C} \mapsto\left(z \mapsto \underline{\omega}_{z}(\underline{C})\right)$ is surjective.

Proof. Let $\pi_{0}$ be the GNS representation of $\underline{\mathfrak{A}}$ for $\underline{\omega}_{0}$. Consider the $\mathrm{C}^{*}$ algebra $\pi_{0}(\mathfrak{Z}(\underline{\mathfrak{A}}))$. It is well known that this commutative algebra is isomorphic to $\mathcal{C}(\mathcal{Z})$ for a compact Hausdorff space $\mathcal{Z}$, with the isomorphism being given by $\pi_{0}(\underline{C}) \mapsto\left(z \mapsto \rho_{z}\left(\pi_{0}(\underline{C})\right)\right)$, where the $\rho_{z}$ are multiplicative functionals. Now by the Riesz representation theorem, the GNS state $\left(\Omega_{0}|\cdot| \Omega_{0}\right)$ on $\pi_{0}(\mathfrak{Z}(\underline{\mathfrak{A}})) \cong \mathcal{C}(\mathcal{Z})$ is given by a regular Borel measure $\nu$ on $\mathcal{Z}$. Explicitly, one has for all $\underline{C} \in \mathfrak{Z}(\underline{\mathfrak{A}})$,

$$
\underline{\omega}_{0}(\underline{C})=\left(\Omega_{0}\left|\pi_{0}(\underline{C})\right| \Omega_{0}\right)=\int_{\mathcal{Z}} d \nu(z) \rho_{z} \circ \pi_{0}(\underline{C}) .
$$

It is clear that $\nu(\mathcal{Z})=1$. In the above expression, $\mathrm{m}_{z}:=\rho_{z} \circ \pi_{0}$ are multiplicative means; they are asymptotic, since $\pi_{0}(\underline{A})=0$ whenever $\underline{A}_{\lambda}$ vanishes for small $\lambda$. Thus, setting $\underline{\omega}_{z}=\rho_{z} \circ \pi_{0} \circ \omega$ as usual, we obtain multiplicative limit states $\underline{\omega}_{z}$ on $\underline{\mathfrak{A}}$ such that

$$
\underline{\omega}_{0}(\underline{A})=\int_{\mathcal{Z}} d \nu(z) \underline{\omega}_{z}(\underline{A}) \quad \text { for all } \underline{A} \in \underline{\mathfrak{A}} \text {. }
$$

As a last point, the map $\mathfrak{Z}(\underline{\mathfrak{A}}) \rightarrow \mathcal{C}(\mathcal{Z}), \underline{C} \mapsto\left(z \mapsto \underline{\omega}_{z}(\underline{C})\right)=\left(z \mapsto \rho_{z}\left(\pi_{0}(\underline{C})\right)\right)$ is surjective by construction. 
We have thus decomposed a general limit state $\underline{\omega}_{0}$ into multiplicative limit states $\underline{\omega}_{z}$. In the case $s \geq 2$, this will also be a decomposition into pure states; but the above result does not depend on that. Also, we emphasize that our aim is not a decomposition of the von Neumann algebra $\pi_{0}(\underline{\mathfrak{A}})^{\prime \prime}$ along its center; rather we work on the $\mathrm{C}^{*}$ algebraic side only.

We would now like to interpret the above decomposition in the sense of decomposing the limit Hilbert space $\mathcal{H}_{0}$ as a direct integral. This is complicated by the fact that our measure spaces $(\mathcal{Z}, \nu)$ can be of a very general nature, making the limit Hilbert space nonseparable. In fact, if $\underline{\omega}_{0}$ is an invariant limit state, one finds that all vectors of the form $\pi_{0}(\underline{C}) \Omega_{0}$ are mutually orthogonal if $\underline{C}_{\lambda}=\chi(\lambda) \mathbf{1}$, where $\chi$ is a character on $\mathbb{R}_{+}$. Since there are clearly uncountably many characters - just take $\chi(\lambda)=\lambda^{\imath k}$ with $k \in \mathbb{R}$ - the limit Hilbert space $\mathcal{H}_{0}$ cannot be separable in this case.

The theory of direct integrals of Hilbert spaces in the absence of separability assumptions is nonstandard and only partially complete; we give a brief review in Appendix A. Here we note that the notion of a direct integral over $\mathcal{Z}$, with fiber spaces $\mathcal{H}_{z}$, crucially depends on the specification of a fundamental family $\Gamma \subset \prod_{z \in \mathcal{Z}} \mathcal{H}_{z}$. This $\Gamma$ is a vector space with certain extra conditions (see Def. A.1) that serves to define which Hilbert space valued functions are considered measurable. Indeed, using the exact notions, we prove:

Theorem 3.2. Let $\underline{\omega}_{0}$ be a limit state, and $\mathcal{Z}, \nu, \underline{\omega}_{z}$ as in Proposition 3.1. Let $\pi_{z}, \mathcal{H}_{z}, \Omega_{z}$ be the GNS representation objects corresponding to $\underline{\omega}_{z}$. Then,

$$
\Gamma:=\left\{z \mapsto \pi_{z}(\underline{A}) \Omega_{z} \mid \underline{A} \in \underline{\mathfrak{A}}\right\} \subset \prod_{z \in \mathcal{Z}} \mathcal{H}_{z}
$$

is a fundamental family. With respect to this family, it holds that

$$
\mathcal{H}_{0} \cong \int_{\mathcal{Z}}^{\Gamma} d \nu(z) \mathcal{H}_{z}
$$

where the isomorphism is given by

$$
\pi_{0}(\underline{A}) \Omega_{0} \mapsto \int_{\mathcal{Z}}^{\Gamma} d \nu(z) \pi_{z}(\underline{A}) \Omega_{z}, \quad \underline{A} \in \underline{\mathfrak{A}} .
$$

Proof. It is clear that $\Gamma$ is a linear space; and per Prop. 3.1, the function $z \mapsto\left\|\pi_{z}(\underline{A}) \Omega_{z}\right\|^{2}=$ $\underline{\omega}_{z}\left(\underline{A}^{*} \underline{A}\right)$ is integrable for any $\underline{A} \in \underline{\mathfrak{A}}$. Thus $\Gamma$ is a fundamental family per Definition A.1. The map

$$
W: \mathcal{H}_{0} \rightarrow \prod_{z \in \mathcal{Z}} \mathcal{H}_{z}, \quad \pi_{0}(\underline{A}) \Omega_{0} \mapsto\left(z \mapsto \pi_{z}(\underline{A}) \Omega_{z}\right)
$$

is clearly linear and isometric when $\underline{A}$ ranges through $\underline{\mathfrak{A}}$; thus $W$ can in fact be extended to a well-defined isometric map from $\mathcal{H}_{0}$ into $\bar{\Gamma}$. It remains to show that $W$ is surjective. In fact, since $L^{\infty}(\mathcal{Z}) \cdot \Gamma$ is total in the direct integral space, it suffices to show that all vectors of the form

$$
\int_{\mathcal{Z}}^{\Gamma} d \nu(z) f(z) \pi_{z}(\underline{A}) \Omega_{z}, \quad f \in L^{\infty}(\mathcal{Z}), \underline{A} \in \underline{\mathfrak{A}},
$$

can be approximated in norm with vectors of the form $W \pi_{0}(\underline{B}) \Omega_{0}, \underline{B} \in \underline{\mathfrak{A}}$.

To that end, let $f \in L^{\infty}(\mathcal{Z})$ and $\underline{A} \in \underline{\mathfrak{A}}$ be fixed. We first note that, as a simple consequence of Lusin's theorem, there exist functions $f_{n} \in \mathcal{C}(\mathcal{Z})$ such that $\left\|f_{n}\right\|_{\infty} \leq\|f\|_{\infty}$ 
and $\lim _{n \rightarrow \infty} f_{n}(z)=f(z)$ for almost every $z \in \mathcal{Z}$. On the other hand, per Proposition 3.1 there exist $\underline{C}_{n} \in \mathfrak{Z}(\underline{\mathfrak{A}})$ such that $\underline{\omega}_{z}\left(\underline{C}_{n}\right)=f_{n}(z)$ for all $z \in \mathcal{Z}$, which implies $\pi_{z}\left(\underline{C}_{n}\right)=f_{n}(z) \mathbf{1}$. Therefore we have

$$
\lim _{n \rightarrow \infty} \int_{\mathcal{Z}}\left\|\left(f(z) \pi_{z}(\underline{A})-\pi_{z}\left(\underline{C}_{n} \underline{A}\right)\right) \Omega_{z}\right\|^{2} d \nu(z)=0
$$

by an application of the dominated convergence theorem.

In the following, we will usually not denote the above isomorphism explicitly, but rather identify $\mathcal{H}_{0}$ with its direct integral representation. In this way, the subspace $\mathcal{H}_{\mathfrak{Z}} \subset \mathcal{H}_{0}$ is isomorphic to the function space $L^{2}(\mathcal{Z}, \nu)$, where $f \in L^{2}(\mathcal{Z}, \nu)$ is identified with $\int_{\mathcal{Z}}^{\Gamma} d \nu(z) f(z) \Omega_{z} \in$ $\mathcal{H}_{0}$. The next corollary follows directly from the proof above, since a decomposition of operators needs to be checked on the fundamental family only (Lemma A.2).

Corollary 3.3. With respect to the direct integral decomposition in Theorem 3.2, all operators $\pi_{0}(\underline{A}), \underline{A} \in \underline{\mathfrak{A}}$ are decomposable, and one has $\pi_{0}=\int_{\mathcal{Z}}^{\Gamma} d \nu(z) \pi_{z}$. If $\underline{A} \in \mathfrak{Z}(\underline{\mathfrak{A}})$, then $\pi_{0}(\underline{A})$ is diagonal, with $\pi_{0}(\underline{A})=\int_{\mathcal{Z}}^{\Gamma} d \nu(z) \underline{\omega}_{z}(\underline{A}) \mathbf{1}$.

Finally, we remark that Lorentz symmetries $U_{0}(x, \Lambda)$ in the limit theory are decomposable.

Proposition 3.4. Let $g \mapsto U_{z}(g)$ be the implementation of $\mathcal{P}_{+}^{\uparrow}$ on the limit Hilbert space $\mathcal{H}_{z}$ corresponding to $\underline{\omega}_{z}$. Then, one has

$$
U_{0}(g)=\int_{\mathcal{Z}}^{\Gamma} d \nu(z) U_{z}(g) \quad \text { for all } g \in \mathcal{P}_{+}^{\uparrow}
$$

Proof. Again, it suffices to verify this on vectors from $\Gamma$. With $W$ being the isomorphism introduced in the proof of Theorem [3.2, one obtains for all $g \in \mathcal{P}_{+}^{\uparrow}$ and $\underline{A} \in \underline{\mathfrak{A}}$,

$$
W U_{0}(g) \pi_{0}(\underline{A}) \Omega_{0}=W \pi_{0}\left(\underline{\alpha}_{g} \underline{A}\right) \Omega_{0}=\int_{\mathcal{Z}}^{\Gamma} d \nu(z) \pi_{z}\left(\underline{\alpha}_{g} \underline{A}\right) \Omega_{z}=\int_{\mathcal{Z}}^{\Gamma} d \nu(z) U_{z}(g) \pi_{z}(\underline{A}) \Omega_{z} .
$$

This proves the proposition.

It should be remarked that the same simple structure cannot be expected for dilations, if they exist as a symmetry of the limit. For even if $\underline{\omega}_{0} \circ \underline{\alpha}_{\mu}=\underline{\omega}_{0}$, the multiplicative limit states $\underline{\omega}_{z}$ cannot be invariant under $\underline{\alpha}_{\mu}$, not even when restricted to $\mathfrak{Z}(\underline{\mathfrak{A}})$. Thus, the unitaries $U_{0}(\mu)$ will not commute with $\pi_{0}(\mathfrak{Z}(\underline{\mathfrak{A}}))$, and can therefore not be decomposable. In special situations, there may be a generalized sense in which the dilation unitaries can be decomposed; we will investigate this in more detail in Sec. [5.

\section{Unique and factorizing scaling limits}

The limit theory on the Hilbert space $\mathcal{H}_{0}$ is composed, as discussed in the previous section, of simpler components that live on the "fibre" Hilbert spaces $\mathcal{H}_{z}$ of the direct integral. It is natural to ask whether the theories on these spaces $\mathcal{H}_{z}$, or more precisely, the nets of algebras $\mathfrak{A}_{z}(\mathcal{O})=\pi_{z}(\mathfrak{A}(\mathcal{O}))^{\prime \prime}$, are similar or identical in a certain sense. While no models have been explicitly constructed for which the limit theories substantially depend on the choice of a 
(multiplicative) limit state 3 it does not seem to be excluded that measurable properties, such as the mass spectrum or charge structure of $\mathfrak{A}_{z}$, can depend on $z$.

For most applications in physics, however, one expects that the situation is simpler, and that the limit theory does not depend substantially on the choice of $\underline{\omega}_{z}$. Here it would be much too strict to require that the representations $\pi_{z}$ are unitarily equivalent. [In fact, for $s \geq 2$, the $\pi_{z}$ are irreducible per Thm. 2.3, and since they do not agree on $\mathfrak{Z}(\underline{\mathfrak{A}})$, they are even pairwise disjoint.] Rather one can expect that their images, the algebras $\mathfrak{A}_{z}(\mathcal{O})$, are unique as sets, up to unitaries that identify the different Hilbert spaces $\mathcal{H}_{z}$; see Def. 4.1 below. This is the situation of a unique scaling limit in the sense of Buchholz and Verch.

In the present section, we want to elaborate how the situation of unique scaling limits, originally formulated for multiplicative limit states, fits into our generalized context. To that end, we will formulate several conditions on the limit theory that roughly correspond to unique limits, and discuss their mutual dependencies.

\subsection{Definitions}

We shall first motivate and define the conditions to be considered; the proofs of their interrelations are deferred to sections further below. We start by recalling the condition of a unique scaling limit in the sense of [BV95], with some slight modifications.

Definition 4.1. The theory $\mathfrak{A}$ is said to have a unique scaling limit if there exists a local Poincaré covariant net $\left(\mathfrak{A}_{\mathrm{u}}, \mathcal{H}_{\mathrm{u}}, \Omega_{\mathrm{u}}, U_{\mathrm{u}}\right)$ in the vacuum sector such that the following holds. For every multiplicative limit state $\underline{\omega}_{0}$, there exists a unitary $V: \mathcal{H}_{0} \rightarrow \mathcal{H}_{\mathrm{u}}$ such that $V \Omega_{0}=$ $\Omega_{\mathrm{u}}, V U_{0}(g) V^{*}=U_{\mathrm{u}}(g)$ for all $g \in \mathcal{P}_{+}^{\uparrow}$, and $V \mathfrak{A}_{0}(\mathcal{O}) V^{*} \subset \mathfrak{A}_{\mathrm{u}}(\mathcal{O})$ for all open bounded regions $\mathcal{O}$.

This includes the aspect of a "unique vacuum structure". Compared with BV95, we have somewhat weakened the condition, since we require only inclusion of $V \mathfrak{A}_{0}(\mathcal{O}) V^{*}$ in $\mathfrak{A}_{\mathrm{u}}(\mathcal{O})$, not equality. This is for the following reason. Supposing that both $\mathfrak{A}$ and $\mathfrak{A}_{\mathrm{u}}$ fulfill the condition of geometric modular action (Definition [2.5), such that the Haag-dualized nets of $\mathfrak{A}_{0}$ and $\mathfrak{A}_{\mathrm{u}}$ are well-defined, our condition precisely implies that these dualized nets agree for all multiplicative limit states. Since for many applications, particularly charge analysis [DMV04], the dualized limit nets are seen as the fundamental objects, we think that this is a reasonable generalization of the condition.

For a general, not necessarily multiplicative limit state $\underline{\omega}_{0}$, we obtain a decomposition $\underline{\omega}_{0}=\int_{\mathcal{Z}} d \nu(z) \underline{\omega}_{z}$ into multiplicative states, as discussed in Sec. 3, and thus obtain from Def. 4.1 corresponding unitaries $V_{z}$ for every $z$. Due to the very general nature of the measure space $\mathcal{Z}$, and due to a possible arbitrariness in the choice of $V_{z}$, particularly if $\mathfrak{A}_{\mathrm{u}}$ possesses inner symmetries, an analysis of $\underline{\omega}_{0}$ seems impossible in this generality. Rather we will often make use of a regularity condition, which is formulated as follows.

Definition 4.2. Suppose that the theory $\mathfrak{A}$ has a unique scaling limit. We say that a limit state $\underline{\omega}_{0}$ is regular if there is a choice of the unitaries $V_{z}$ such that for any $\underline{A} \in \underline{\mathfrak{A}}$, the function $\varphi_{\underline{A}}: \overline{\mathcal{Z}} \rightarrow \mathcal{H}_{\mathrm{u}}, z \mapsto V_{z} \pi_{z}(\underline{A}) \Omega_{z}$ is Lusin measurable [i.e., is contained in $\left.\bar{L}^{2}\left(\mathcal{Z}, \nu, \mathcal{H}_{\mathrm{u}}\right)\right]$.

We shall later give a sufficient condition for the above regularity, which actually implies that the functions $\varphi_{\underline{A}}$ in fact be chosen constant in generic cases.

\footnotetext{
${ }^{3}$ See however [Buc96a, Sec. 5] for some ideas to that end.
} 
Our concepts so far refer to multiplicative limit states mostly. We will now give a generalization of Def. 4.1 that involves generalized limit states directly, and that seems natural in our context. It is based on the picture that the limit Hilbert space should have a tensor product structure, $\mathcal{H}_{0} \cong \mathcal{H}_{\mathfrak{Z}} \otimes \mathcal{H}_{\mathrm{u}}$, where $\mathcal{H}_{\mathrm{u}}$ is the unique representation space associated with multiplicative limit states, and $\mathcal{H}_{\mathfrak{Z}}$ is the representation space of $\mathfrak{Z}(\underline{\mathfrak{A}})$ under $\pi_{0}$. All objects of the theory - local algebras, Poincaré symmetries, and the vacuum vector - should factorize along this tensor product. We now formulate this in detail.

Definition 4.3. The theory $\mathfrak{A}$ is said to have a factorizing scaling limit if there exists a local Poincaré covariant net $\left(\mathfrak{A}_{\mathrm{u}}, \mathcal{H}_{\mathrm{u}}, \Omega_{\mathrm{u}}, U_{\mathrm{u}}\right)$ in the vacuum sector such that the following holds. For every limit state $\underline{\omega}_{0}$, there exists a decomposable unitary $V: \mathcal{H}_{0} \rightarrow L^{2}\left(\mathcal{Z}, \nu, \mathcal{H}_{\mathrm{u}}\right), V=$ $\int_{\mathcal{Z}}^{\Gamma, \oplus} d \nu(z) V_{z}$ with unitaries $V_{z}: \mathcal{H}_{z} \rightarrow \mathcal{H}_{\mathrm{u}}$, such that $V \Omega_{0}=\Omega_{\mathfrak{Z}} \otimes \Omega_{\mathrm{u}}, V U_{0}(g) V^{*}=\mathbf{1} \otimes U_{\mathrm{u}}(g)$ for all $g \in \mathcal{P}_{+}^{\uparrow}$, and $V_{z} \mathfrak{A}_{z}(\mathcal{O}) V_{z}^{*} \subset \mathfrak{A}_{\mathrm{u}}(\mathcal{O})$ for all open bounded regions $\mathcal{O}$ and all $z \in \mathcal{Z}$.

Here $\Omega_{\mathfrak{Z}} \in \mathcal{H}_{\mathfrak{Z}}$ denotes the GNS vector of the commutative algebra. The conditions on local algebras are deliberately chosen quite strict. We require $V_{z} \mathfrak{A}_{z}(\mathcal{O}) V_{z}^{*} \subset \mathfrak{A}_{\mathrm{u}}(\mathcal{O})$ for every $z$, rather than the weaker condition $V \mathfrak{A}_{0}(\mathcal{O}) V^{*} \subset \mathfrak{Z}_{0} \bar{\otimes} \mathfrak{A}_{\mathrm{u}}(\mathcal{O})$. This serves to avoid countability problems; see Sec. 4.3 for further discussion.

In subsequent sections, we will show that the notion of a unique scaling limit and a factorizing scaling limit are cum grano salis identical, up to the extra regularity condition in Definition 4.2 that we have to assume.

We also consider a stronger condition, which is easier to check in models. Our ansatz is

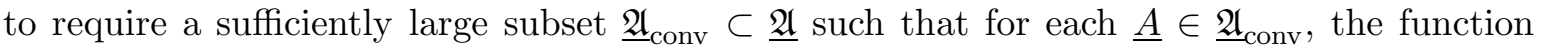
$\lambda \mapsto \omega\left(\underline{A}_{\lambda}\right)$ is convergent as $\lambda \rightarrow 0$. Consider the following definition:

Definition 4.4. The theory $\mathfrak{A}$ is said to have a convergent scaling limit if there exists an $\underline{\alpha}$-invariant $C^{*}$ subalgebra $\underline{\mathfrak{A}}_{\mathrm{conv}} \subset \underline{\mathfrak{A}}$ with the following properties:

(i) For each $\underline{A} \in \underline{\mathfrak{A}}_{\mathrm{conv}}$, the function $\lambda \mapsto \omega\left(\underline{A}_{\lambda}\right)$ converges as $\lambda \rightarrow 0$.

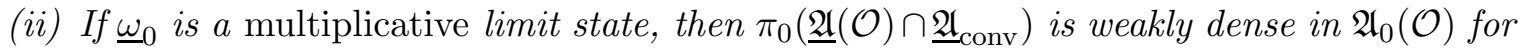
every open bounded region $\mathcal{O}$.

It follows directly from (ii) that also $\pi_{0}\left(\underline{\mathfrak{A}}_{\text {conv }}\right) \Omega_{0}$ is dense in $\mathcal{H}_{0}$. The condition roughly says that "convergent scaling functions" are sufficient for describing the limit theory - considering nonconvergent sequences is only required for technical consistency of our formalism, for describing the image of $\mathfrak{Z}(\underline{\mathfrak{A}})$, which does not directly relate to quantum theory. This is heuristically expected in many physical models: In usual renormalization approaches in formal perturbation theory, the selection of subsequences or filters to enforce convergence seems not to be widespread, and sequences of pointlike fields can be chosen to converge in matrix elements.

We will show that the above condition is sufficient for the scaling limit to be unique, and all limit states to be regular. In fact, we shall see later that also the structure of dilations simplifies.

Figure 1 summarizes the different conditions we introduced, and shows the implications we briefly mentioned. We will now go ahead and prove that the individual arrows are indeed correct. However, in order to avoid problems with the direct integral spaces involved, we shall make certain separability assumptions in most cases. Let us comment on these. For multiplicative limit states, it seems a reasonable assumption that the limit Hilbert space $\mathcal{H}_{0}$ 
Figure 1: Implications between the conditions on the limit theory. Arrows marked with $*$ are only proven under additional separability assumptions.

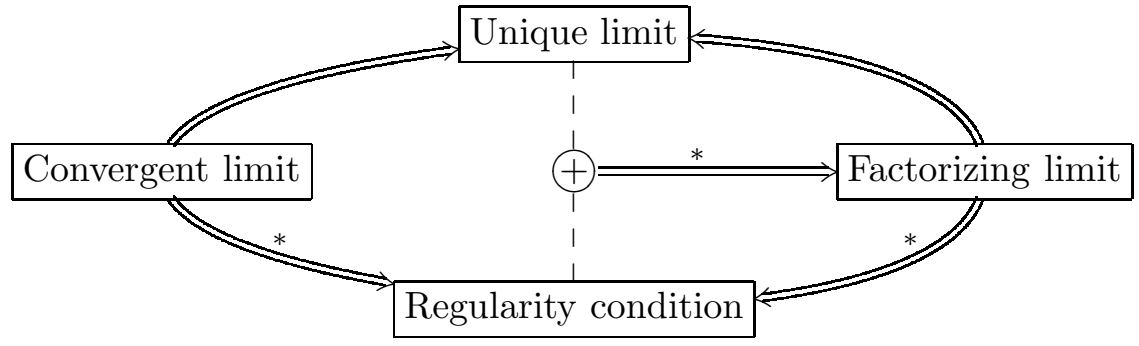

is separable. This would follow, from example, from the Haag-Swieca compactness condition; cf. Buc96a]. For general limit states, in particular if these are invariant, $\mathcal{H}_{0}$ cannot be separable since already $\mathcal{H}_{\mathfrak{Z}} \cong L^{2}(\mathcal{Z}, \nu)$ is nonseparable, as discussed in Sec. 3. We can however reasonably assume that $\mathcal{H}_{0}$ fulfills a condition which we call uniform separability; cf. Def. A.3 in the appendix. This means that a countable set $\left\{\chi_{j}\right\} \subset \mathcal{H}_{0}=\int_{\mathcal{Z}}^{\Gamma} d \nu(z) \mathcal{H}_{z}$ exists such that $\left\{\chi_{j}(z)\right\}$ is dense in every $\mathcal{H}_{z}$. As we shall see in Sec. 6, uniform separability follows from a sharpened version of the Haag-Swieca compactness condition; and we will show in Sec. 7 that this compactness condition is indeed fulfilled in relevant examples.

\subsection{Unique limit $\Rightarrow$ factorizing limit}

In the following, we suppose that $\mathfrak{A}$ has a unique scaling limit. We fix a regular limit state $\underline{\omega}_{0}$, and denote the associated objects $\mathcal{Z}, \nu, \mathcal{H}_{0}, \pi_{0}, \Omega_{0}, \mathcal{H}_{z}, \pi_{z}, \Omega_{z}, V_{z}$ as usual. In order to prove that the scaling limit factorizes, we have to construct a unitary $V: \mathcal{H}_{0} \rightarrow L^{2}\left(\mathcal{Z}, \nu, \mathcal{H}_{\mathrm{u}}\right)$ with appropriate properties. In fact, this $V$ is intuitively given by $V=\int_{\mathcal{Z}}^{\Gamma, \oplus} d \nu(z) V_{z}$; and the key question turns out to be whether this $V$ is surjective. We will prove this only under separability assumptions.

Proposition 4.5. Let $\mathfrak{A}$ have a unique scaling limit; let $\underline{\omega}_{0}$ be a regular limit state; and suppose that $\mathcal{H}_{0}$ is uniformly separable. Then,

$$
V: \mathcal{H}_{0} \rightarrow L^{2}\left(\mathcal{Z}, \nu, \mathcal{H}_{\mathrm{u}}\right), \quad V=\int_{\mathcal{Z}}^{\Gamma, \oplus} d \nu(z) V_{z}
$$

defines a unitary operator.

Proof. First, it is clear that if $\mathcal{H}_{0}$ is uniformly separable, then all $\mathcal{H}_{z}$, and in particular $\mathcal{H}_{\mathrm{u}}$, are separable. Hence $L^{2}\left(\mathcal{Z}, \nu, \mathcal{H}_{\mathrm{u}}\right)$ is uniformly separable.

Now note that $V$ is well-defined precisely by the regularity condition. Further, writing explicitly

$$
V \pi_{0}(\underline{A}) \Omega_{0}=\left(z \mapsto V_{z} \pi_{z}(\underline{A}) \Omega_{z}\right), \quad \underline{A} \in \underline{\mathfrak{A}},
$$

one has

$$
\left\|V \pi_{0}(\underline{A}) \Omega_{0}\right\|^{2}=\int_{\mathcal{Z}} d \nu(z)\left\|V_{z} \pi_{z}(\underline{A}) \Omega_{z}\right\|^{2}=\int_{\mathcal{Z}} d \nu(z)\left\|\pi_{z}(\underline{A}) \Omega_{z}\right\|^{2}=\left\|\pi_{0}(\underline{A}) \Omega_{0}\right\|^{2},
$$


so $V$ is isometric. It remains to show that $V$ is surjective. To that end, let $P$ be the orthogonal projector onto $\operatorname{img} V$. Since $V$ commutes with all diagonal operators, so does $P$; thus $P$ is decomposable: $P=\int_{\mathcal{Z}}^{\oplus} d \nu(z) P(z)$. Now compute

$$
0=(1-P) V=\int_{\mathcal{Z}}^{\Gamma, \oplus} d \nu(z)(1-P(z)) V_{z}
$$

Using uniform separability of both spaces involved, we obtain that $(1-P(z)) V_{z}=0$ a. e. Since the $V_{z}$ are surjective onto $\mathcal{H}_{\mathrm{u}}$, this means $P(z)=\mathbf{1}$ a. e. This implies $P=\mathbf{1}$, so $V$ is surjective.

It is clear that $V \Omega_{0}=\Omega_{\mathfrak{Z}} \otimes \Omega_{\mathrm{u}}$; and we can also verify from the properties of the $V_{z}$ with respect to Poincaré symmetries that

$$
V U_{0}(g) V^{*}=\mathbf{1} \otimes U_{\mathbf{u}}(g) \text { for all } g \in \mathcal{P}_{+}^{\uparrow} .
$$

Also, by the definition of the unique scaling limit, it must hold that $V_{z} \mathfrak{A}_{z}(\mathcal{O}) V_{z}^{*} \subset \mathfrak{A}_{\mathrm{u}}(\mathcal{O})$ for all $z$. Summarizing the results of this section, we have shown:

Theorem 4.6. Suppose that $\mathfrak{A}$ has a unique scaling limit, that every limit state $\underline{\omega}_{0}$ is regular, and that the limit spaces $\mathcal{H}_{0}$ are uniformly separable. Then the scaling limit of $\mathfrak{A}$ is factorizing.

\subsection{Factorizing limit $\Rightarrow$ unique limit}

Now reversing the arrow, we start from a theory with factorizing scaling limit, and want to show that the scaling limit is unique in the sense of Buchholz and Verch, and that the limit states are regular. At first glance, this implication seems to be apparent from the definitions. A detailed investigation however reveals some subtleties, which again lead us to making separability assumptions.

Theorem 4.7. Assume that $\mathfrak{A}$ has a factorizing scaling limit. Then the scaling limit is unique. If the space $\mathcal{H}_{\mathrm{u}}$ is separable, all limit states $\underline{\omega}_{0}$ are regular.

Proof. It is clear that the scaling limit is unique by Def. 4.1, specializing the conditions of Def. 4.3 to the case where $\underline{\omega}_{0}$ is multiplicative, and $\mathcal{Z}$ consists of a single point. Now let $\underline{\omega}_{0}$ be a limit state; we need to show it is regular. Let $V=\int_{\mathcal{Z}}^{\Gamma, \oplus} d \nu(z) V_{z}$ be the unitary guaranteed by Def. 4.3. By definition, the map $z \mapsto V_{z} \pi_{z}(\underline{A}) \Omega_{z}$ is measurable for any $A \in \underline{A}$. But we have to show that each $V_{z}$ fulfills the conditions of Def. 4.1) in fact, we will have to modify the $V_{z}$ on a null set.

First, we have $V \Omega_{0}=\Omega_{\mathfrak{Z}} \otimes \Omega_{\mathrm{u}}$ by assumption. On the other hand, $V \Omega_{0}=\int_{\mathcal{Z}}^{\oplus} d \nu(z) V_{z} \Omega_{z}$, so that $V_{z} \Omega_{z}=\Omega_{\mathrm{u}}$ for $z \in \mathcal{Z} \backslash \mathcal{N}_{\Omega}$, where $\mathcal{N}_{\Omega}$ is a null set. Next we consider Poincaré transformations. Starting from Def. 4.3, we know that:

$$
V U_{0}(g) V^{*}=\mathbf{1} \otimes U_{\mathbf{u}}(g) \text { for all } g \in \mathcal{P}_{+}^{\uparrow} .
$$

Since $U_{0}(g)$ factorizes by Prop. 3.4, we can rewrite this equation as

$$
\int_{\mathcal{Z}}^{\oplus} d \nu(z) V_{z} U_{z}(g) V_{z}^{*}=\int_{\mathcal{Z}}^{\oplus} d \nu(z) U_{\mathbf{u}}(g) .
$$


Now if $\mathcal{H}_{\mathrm{u}}$ is separable, and thus $L^{2}\left(\mathcal{Z}, \nu, \mathcal{H}_{\mathrm{u}}\right)$ uniformly separable, we can conclude that $V_{z} U_{z}(g) V_{z}^{*}=U_{\mathrm{u}}(g)$ for all $z \in \mathcal{Z} \backslash \mathcal{N}_{g}$, with a null set $\mathcal{N}_{g}$ depending on $g$. We pick a countable dense subset $\mathcal{P}_{c}$ of $\mathcal{P}_{+}^{\uparrow}$, and consider the null set $\mathcal{N}:=\mathcal{N}_{\Omega} \cup\left(\cup_{g \in \mathcal{P}_{c}} \mathcal{N}_{g}\right)$. Our results so far are that

$$
V_{z} \Omega_{z}=\Omega_{\mathrm{u}}, \quad V_{z} U_{z}(g) V_{z}^{*}=U_{\mathrm{u}}(g) \quad \text { for all } z \in \mathcal{Z} \backslash \mathcal{N}, g \in \mathcal{P}_{c} .
$$

Indeed, by continuity of the representations, the same holds for all $g \in \mathcal{P}_{+}^{\uparrow}$. Now let $\hat{V}_{z}$ be those unitaries obtained by evaluating Def. 4.3 for the multiplicative limit states $\underline{\omega}_{z}$. We set

$$
W_{z}:= \begin{cases}V_{z} & \text { for } z \in \mathcal{Z} \backslash \mathcal{N}, \\ \hat{V}_{z} & \text { for } z \in \mathcal{N} .\end{cases}
$$

Then we have $V=\int_{\mathcal{Z}}^{\Gamma, \oplus} d \nu(z) W_{z}$, and the $W_{z}$ fulfill the relations in Eq. (4.7) for all $z \in \mathcal{Z}$ and $g \in \mathcal{P}_{+}^{\uparrow}$. As a last point, $W_{z} \mathfrak{A}_{z}(\mathcal{O}) W_{z}^{*} \subset \mathfrak{A}_{\mathrm{u}}(\mathcal{O})$ holds for every $z$, since both $V_{z}$ and $\hat{V}_{z}$ have this property. Thus $\underline{\omega}_{0}$ is regular.

Let us add some comments on the conditions required for $V_{z}$ in Def.4.3, regarding Poincaré transformations and local algebras. We could choose stricter conditions on $V_{z}$, requiring that

$$
V_{z} U_{z}(g) V_{z}^{*}=U_{\mathrm{u}}(g) \text { for all } z \in \mathcal{Z} \text { and } g \in \mathcal{P}_{+}^{\uparrow} .
$$

In this case, the countability problem in the proof above does not occur, and Thm. 4.7 holds without the requirement that $\mathcal{H}_{\mathrm{u}}$ is separable.

On the other hand, it does not seem reasonable to weaken the conditions on $V_{z}$ with respect to local algebras, requiring only that

$$
V \mathfrak{A}_{0}(\mathcal{O}) V^{*} \subset \mathfrak{Z}_{0} \bar{\otimes} \mathfrak{A}_{\mathrm{u}}(\mathcal{O}) \text { for all } \mathcal{O} \text {. }
$$

(We shall show below that this relation is implied by the chosen conditions on $V_{z}$.) For if we require only (4.10), and we wish to apply the techniques used in the proof of Thm. 4.7, it becomes necessary not only to require separability of $\mathcal{H}_{\mathrm{u}}$ - which seems reasonable for applications in physics -, but also separability of the algebras $\mathfrak{A}_{\mathbf{u}}(\mathcal{O})$. That would however be too strict for our purposes, since the local algebras are expected to be isomorphic to the hyperfinite type $\mathrm{III}_{1}$ factor $\mathrm{BDF} 87$ ].

We now show that Eq. (4.10) follows from Def. 4.3 as given.

Proposition 4.8. Let $\mathfrak{A}$ have a factorizing scaling limit. With $V$ the unitary of Def. 4.3 , one has $V \mathfrak{A}_{0}(\mathcal{O}) V^{*} \subset \mathfrak{Z}_{0} \bar{\otimes} \mathfrak{A}_{\mathrm{u}}(\mathcal{O})$ for any bounded open region $\mathcal{O}$.

Proof. Let $\underline{A} \in \underline{\mathfrak{A}}(\mathcal{O})$, and $A^{\prime} \in \mathfrak{A}_{\mathrm{u}}(\mathcal{O})^{\prime}$. We compute the commutator $\left[\mathbf{1} \otimes A^{\prime}, V \pi_{0}(\underline{A}) V^{*}\right]$ as a direct integral:

$$
\left[\mathbf{1} \otimes A^{\prime}, V \pi_{0}(\underline{A}) V^{*}\right]=\int_{\mathcal{Z}}^{\oplus} d \nu(z)\left[A^{\prime}, V_{z} \pi_{z}(\underline{A}) V_{z}^{*}\right] .
$$

Now by our requirements on the $V_{z}$, we have $V_{z} \pi_{z}(\underline{A}) V_{z}^{*} \in \mathfrak{A}_{\mathrm{u}}(\mathcal{O})$ for all $z$, hence the commutator under the integral vanishes. Since $\underline{A} \in \underline{\mathfrak{A}}(\mathcal{O})$ was arbitrary, this means

$$
V \pi_{0}(\underline{\mathfrak{A}}(\mathcal{O})) V^{*} \subset\left(\mathbf{1} \otimes \mathfrak{A}_{\mathrm{u}}(\mathcal{O})^{\prime}\right)^{\prime}=\mathfrak{Z}_{0} \bar{\otimes} \mathfrak{A}_{\mathrm{u}}(\mathcal{O}) .
$$

By weak closure, this inclusion extends to $V \mathfrak{A}_{0}(\mathcal{O}) V^{*}$. 


\subsection{Convergent limit $\Rightarrow$ unique limit}

We now assume that the theory has a convergent scaling limit, and show that our other conditions follow. The main simplification in the convergent case is as follows: For every $\underline{A} \in \underline{\mathfrak{A}}_{\text {conv }}$, the function $\lambda \mapsto \omega\left(\underline{A}_{\lambda}\right)$ converges to a finite limit as $\lambda \rightarrow 0$; so all asymptotic means applied to this function yield the same value. Hence the value of $\underline{\omega}_{0}(\underline{A})$ is the same for all limit states $\underline{\omega}_{0}$, multiplicative or not.

Theorem 4.9. If the scaling limit of $\mathfrak{A}$ is convergent, then it is unique. If further a multiplicative limit state exists such that the associated limit space $\mathcal{H}_{0}$ is separable, then all limit states are regular, and $\mathcal{H}_{0}$ is uniformly separable for any limit state.

Proof. We pick a fixed multiplicative limit state $\underline{\omega}_{u}$ and denote the corresponding representation objects as $\mathcal{H}_{\mathrm{u}}, \pi_{\mathrm{u}}, U_{\mathrm{u}}, \Omega_{\mathrm{u}}$. Given any other multiplicative limit state $\underline{\omega}_{0}$, we define a map $V$ by

$$
V: \mathcal{H}_{0} \rightarrow \mathcal{H}_{\mathrm{u}}, \quad \pi_{0}(\underline{A}) \Omega_{0} \mapsto \pi_{\mathrm{u}}(\underline{A}) \Omega_{\mathrm{u}} \quad \text { for all } \underline{A} \in \underline{\mathfrak{A}}_{\text {conv }} .
$$

The convergence property of $\underline{A} \in \underline{\mathfrak{A}}_{\text {conv }}$ implies

$$
\left\|\pi_{0}(\underline{A}) \Omega_{0}\right\|^{2}=\underline{\omega}_{0}\left(\underline{A}^{*} \underline{A}\right)=\underline{\omega}_{\mathrm{u}}\left(\underline{A}^{*} \underline{A}\right)=\left\|\pi_{\mathrm{u}}(\underline{A}) \Omega_{\mathrm{u}}\right\|^{2},
$$

so the linear map $V$ is both well-defined and isometric. It is also densely defined and surjective by assumption (Def. 4.4). Hence $V$ extends to a unitary. Using the $\underline{\alpha}$-invariance of $\underline{A}_{\text {conv }}$, one checks by direct computation that $V U_{0}(g) V^{*}=U_{\mathrm{u}}(g)$ for all $g \in \mathcal{P}_{+}^{\uparrow}$. Also, $V \Omega_{0}=\Omega_{\mathrm{u}}$ is clear. Further, if $\underline{A} \in \underline{\mathfrak{A}}(\mathcal{O}) \cap \underline{\mathfrak{A}}_{\text {conv }}$, it is clear that $V \pi_{0}(\underline{A}) V^{*}=\pi_{\mathrm{u}}(\underline{A})$. By weak density, this means $V \mathfrak{A}_{0}(\mathcal{O}) V^{*}=\mathfrak{A}_{0}(\mathcal{O})$. Thus the scaling limit is unique.

Now let $\underline{\omega}_{0}$ not necessarily be multiplicative. Decomposing it into multiplicative states $\underline{\omega}_{z}$ as in Prop. 3.1, the above construction gives us unitaries $V_{z}: \mathcal{H}_{z} \rightarrow \mathcal{H}_{\mathrm{u}}$ for every $z$. In fact, the functions $z \mapsto V_{z} \pi_{z}(\underline{A}) \Omega_{z}=\pi_{\mathrm{u}}(\underline{A}) \Omega_{\mathrm{u}}$ are constant for all $\underline{A} \in \underline{\mathfrak{A}}_{\mathrm{conv}}$, in particular measurable. Now let $\chi \in \mathcal{H}_{\mathrm{u}}$ and $\underline{B} \in \underline{\mathfrak{A}}$. We can choose a sequence $\left(\underline{A}_{n}\right)_{n \in \mathbb{N}}$ in $\underline{\mathfrak{A}}_{\text {conv }}$ such that $\pi_{\mathrm{u}}\left(\underline{A}_{n}\right) \Omega_{\mathrm{u}} \rightarrow \chi$ in norm. Noticing that

$$
\left(V_{z} \pi_{z}(\underline{B}) \Omega_{z} \mid \chi\right)=\lim _{n \rightarrow \infty}\left(V_{z} \pi_{z}(\underline{B}) \Omega_{z} \mid \pi_{\mathrm{u}}\left(\underline{A}_{n}\right) \Omega_{\mathrm{u}}\right)=\lim _{n \rightarrow \infty} \underline{\omega}_{z}\left(\underline{B}^{*} \underline{A}_{n}\right),
$$

we see that the left-hand side, as a function of $z$, is the pointwise limit of continuous functions, and hence measurable. Thus $z \mapsto V_{z} \pi_{z}(\underline{B}) \Omega_{z}$ is weakly measurable. Now if $\mathcal{H}_{\mathrm{u}}$ was chosen separable, which is possible by assumption, weak measurability implies Lusin measurability of the function (cf. Appendix). Thus $\underline{\omega}_{0}$ is regular.

Finally, in the separable case, we remark that we can pick a countable subset of $\underline{\mathfrak{A}}_{\text {count }} \subset$ $\underline{\mathfrak{A}}_{\text {conv }}$ such that $\pi_{\mathrm{u}}\left(\underline{\mathfrak{A}}_{\text {count }}\right) \Omega_{\mathrm{u}}$ is dense in $\mathcal{H}_{\mathrm{u}}$. Then $\pi_{0}\left(\underline{\mathfrak{A}}_{\text {count }}\right) \Omega_{0}$ becomes a fundamental sequence in $\mathcal{H}_{0}$, so that this space is uniformly separable.

Of course, it follows as a corollary to the preceding sections that the limit is also factorizing. Let us spell this out more explicitly.

Proposition 4.10. Suppose that $\mathfrak{A}$ has a convergent scaling limit, and that there exists a multiplicative limit state $\underline{\omega}_{\mathrm{u}}$ for which the representation space $\mathcal{H}_{\mathrm{u}}$ is separable. Let $\underline{\omega}_{0}$ be any scaling limit state. There exists a unitary $V=\int_{\mathcal{Z}}^{\Gamma, \oplus} d \nu(z) V_{z}: \mathcal{H}_{0} \rightarrow L^{2}\left(\mathcal{Z}, \nu, \mathcal{H}_{\mathrm{u}}\right)$ such that

$$
V \pi_{0}(\underline{A} \underline{C}) \Omega_{0}=\pi_{0}(\underline{C}) \Omega_{\mathfrak{Z}} \otimes \pi_{\mathrm{u}}(\underline{A}) \Omega_{\mathrm{u}} \quad \text { for all } \underline{A} \in \underline{\mathfrak{A}}_{\text {conv }}, \underline{C} \in \mathfrak{Z}(\underline{\mathfrak{A}}),
$$

and such that the $V_{z}$ fulfill all requirements of Def. 4.3 . 
Proof. We use notation as in the proof of Thm. 4.9. Let $V_{z}: \mathcal{H}_{z} \rightarrow \mathcal{H}_{\mathrm{u}}$ be the unitaries constructed there. Then, $z \mapsto V_{z}^{*}$ is a measurable family of operators. Namely, for any $\underline{A} \in \underline{\mathfrak{A}}_{\mathrm{conv}}$, we find

$$
V_{z}^{*} \pi_{\mathrm{u}}(\underline{A}) \Omega_{\mathrm{u}}=\pi_{z}(\underline{A}) \Omega_{z}
$$

which is in $\Gamma$; hence measurability is checked on the fundamental family (cf. Lemma A.2). So the operator

$$
V^{*}:=\int_{\mathcal{Z}}^{\oplus, \Gamma} d \nu(z) V_{z}^{*}
$$

is well-defined. Domain and range of $V^{*}$ are both uniformly separable, see Thm. 4.9, Thus also the adjoint of $V^{*}$, denoted as $V$, is decomposable with $V=\int_{\mathcal{Z}}^{\Gamma, \oplus} d \nu(z) V_{z}$. It is then clear that $V$ is unitary. Also, we have for $\underline{A} \in \underline{\mathfrak{A}}_{\mathrm{conv}}$ and $\underline{C} \in \mathfrak{Z}(\underline{\mathfrak{A}})$,

$$
\begin{aligned}
V \pi_{0}(\underline{A} \underline{C}) \Omega_{0}=V \int_{\mathcal{Z}}^{\Gamma} d \nu(z) & \pi_{z}(\underline{C}) \pi_{z}(\underline{A}) \Omega_{z}=\int_{\mathcal{Z}}^{\oplus} d \nu(z) \pi_{z}(\underline{C}) V_{z} \pi_{z}(\underline{A}) \Omega_{z} \\
& =\int_{\mathcal{Z}}^{\oplus} d \nu(z) \pi_{z}(\underline{C}) \pi_{\mathrm{u}}(\underline{A}) \Omega_{\mathrm{u}}=\left(\pi_{0}(\underline{C}) \Omega_{\mathfrak{Z}}\right) \otimes\left(\pi_{\mathrm{u}}(\underline{A}) \Omega_{\mathrm{u}}\right) .
\end{aligned}
$$

As a direct consequence of the discussion following Eq. (4.14), the $V_{z}$ have all the properties required in Def. 4.3 regarding vacuum vector, symmetries, and local algebras.

\section{Dilation covariance in the limit}

Our next aim is to analyze the structure of dilation symmetries in the limit theory. To that end, we consider a scaling limit state $\underline{\omega}_{0}$ which is invariant under $\underline{\delta}_{\mu}$. As shown in BDM09, sec. 2], the associated limit theory is covariant with respect to a strongly continuous unitary representation $g \in \underline{\mathcal{G}} \mapsto U_{0}(g)$ of the extended symmetry group $\underline{\mathcal{G}}$, including both Poincaré symmetries and dilations. Our interest is how the dilation unitaries $U_{0}(\mu)$ relate to decomposition theory in Sec. 3, and how they behave in the more specific situations analyzed in Sec. 4. We will consider three cases of decreasing scope: first, the general situation; second, the factorizing scaling limit; third, the convergent scaling limit.

We first consider a general theory as in Sec. 3, and analyze the decomposition of the dilation operators corresponding to the direct integral decomposition of $\mathcal{H}_{0}$ introduced in Thm. 3.2. To this end, we first note that $\underline{\delta}_{\mu}$ leaves $\mathfrak{Z}(\underline{\mathfrak{A}})$ invariant; thus we have a representation of the dilations $U_{\mathfrak{Z}}(\mu):=U_{0}(\mu)\left\lceil\mathcal{H}_{\mathfrak{Z}}\right.$ on $\mathcal{H}_{\mathfrak{Z}}$. Identifying $\mathcal{H}_{\mathfrak{Z}}$ with $L^{2}(\mathcal{Z}, \nu)$ as before, the $U_{\mathfrak{Z}}(\mu)$ act on a function space. This action, and its extension to the entire Hilbert space, can be described in more detail.

Proposition 5.1. Let $\underline{\omega}_{0}$ be an invariant limit state. There exist an action of the dilations through homeomorphisms $z \mapsto \mu . z$ of $\mathcal{Z}$, and unitary operators $U_{z}(\mu): \mathcal{H}_{z} \rightarrow \mathcal{H}_{\mu . z}$ for $\mu \in \mathbb{R}_{+}$, $z \in \mathcal{Z}$, such that:

(i) the measure $\nu$ is invariant under the transformation $z \mapsto \mu . z$;

(ii) $\left(U_{\mathfrak{Z}}(\mu) \chi\right)(z)=\chi\left(\mu^{-1} . z\right)$ for all $\chi \in L^{2}(\mathcal{Z}, \nu)$, as an equation in the $L^{2}$ sense;

(iii) $U_{z}(1)=1, U_{z}(\mu)^{*}=U_{\mu . z}\left(\mu^{-1}\right), U_{\mu . z}\left(\mu^{\prime}\right) U_{z}(\mu)=U_{z}\left(\mu^{\prime} \mu\right)$ for all $z \in \mathcal{Z}, \mu, \mu^{\prime} \in \mathbb{R}_{+}$;

(iv) $U_{z}(\mu) U_{z}(x, \Lambda)=U_{\mu . z}(\mu x, \Lambda) U_{z}(\mu)$ for all $z \in \mathcal{Z},(x, \Lambda) \in \mathcal{P}_{+}^{\uparrow}$; 
(v) $U_{0}(\mu) \chi=\int_{\mathcal{Z}}^{\Gamma} d \nu(z) U_{\mu^{-1} . z}(\mu) \chi\left(\mu^{-1} . z\right)$ for all $\chi \in \int_{\mathcal{Z}}^{\Gamma} d \nu(z) \mathcal{H}_{z}$.

Proof. Recalling that $\mathcal{Z}$ is the spectrum of the commutative $\mathrm{C}^{*}$ algebra $\pi_{0}(\mathfrak{Z}(\underline{\mathfrak{A}}))$, we define the homeomorphism $z \mapsto \mu . z$ as the one induced by the automorphism ad $U_{\mathfrak{Z}}\left(\mu^{-1}\right)$ of $\pi_{0}(\mathfrak{Z}(\underline{\mathfrak{A}}))$. For $\underline{C} \in \mathfrak{Z}(\underline{\mathfrak{A}})$, we know that $\pi_{0}(\underline{C}) \Omega_{0} \in \mathcal{H}_{\mathfrak{Z}}$ corresponds to the function $\chi_{\underline{C}}(z)=\underline{\omega}_{z}(\underline{C})$, precisely the image of $\pi_{0}(\underline{C})$ in the Gelfand isomorphism. Applying $U_{0}\left(\mu^{-1}\right)$ to this vector, one obtains

$$
\left(U_{\mathfrak{Z}}\left(\mu^{-1}\right) \chi_{\underline{C}}\right)(z)=\chi_{\underline{C}}(\mu . z)
$$

thus (ii) holds for all $\chi \in \mathcal{C}(\mathcal{Z})$. Taking the scalar product of Eq. (5.1) with $\Omega_{0}$, one sees that $\int_{\mathcal{Z}} d \nu(z) \chi(z)=\int_{\mathcal{Z}} d \nu(z) \chi(\mu . z)$ for all $\mu$ and $\chi \in \mathcal{C}(\mathcal{Z})$, so (i) follows. Now for general $\chi \in L^{2}(\mathcal{Z}, \nu)$, statement (ii) follows by density.

Translating the action of $z \mapsto \mu . z$ to the level of algebras, it is easy to see that

$$
\underline{\omega}_{\mu . z} \circ \underline{\delta}_{\mu}\left\lceil\mathfrak{Z}(\underline{\mathfrak{A}})=\underline{\omega}_{z}\lceil\mathfrak{Z}(\underline{\mathfrak{A}}) .\right.
$$

Since however $\underline{\delta}_{\mu}$ commutes with the projector $\omega: \underline{\mathfrak{A}} \rightarrow \mathfrak{Z}(\underline{\mathfrak{A}})$, the same equation holds on all of $\underline{\mathfrak{A}}$. Therefore, the maps $U_{z}(\mu): \mathcal{H}_{z} \rightarrow \mathcal{H}_{\mu . z}$ given by

$$
U_{z}(\mu) \pi_{z}(\underline{A}) \Omega_{z}:=\pi_{\mu . z}\left(\underline{\delta}_{\mu}(\underline{A})\right) \Omega_{\mu . z}
$$

are well-defined and unitary. The properties of $U_{z}(\mu)$ listed in (iii) and (iv) then follow from this definition by easy computations.

Now for (v) As before, we identify $\mathcal{H}_{0}$ with $\int_{\mathcal{Z}}^{\Gamma} d \nu(z) \mathcal{H}_{z}$. Then we have, for all $\underline{A} \in \underline{\mathfrak{A}}$,

$$
\begin{aligned}
U_{0}(\mu) \pi_{0}(\underline{A}) \Omega_{0} & =\pi_{0}\left(\underline{\delta}_{\mu}(\underline{A})\right) \Omega_{0}=\int_{\mathcal{Z}}^{\Gamma} d \nu(z) \pi_{z}\left(\underline{\delta}_{\mu}(\underline{A})\right) \Omega_{z} \\
& =\int_{\mathcal{Z}}^{\Gamma} d \nu(z) U_{\mu^{-1} . z}(\mu) \pi_{\mu^{-1} . z}(\underline{A}) \Omega_{\mu^{-1} . z} .
\end{aligned}
$$

Given now a vector $\chi \in \int_{\mathcal{Z}}^{\Gamma} d \nu(z) \mathcal{H}_{z}$, we can find a sequence $\left(\pi_{0}\left(\underline{A}_{n}\right) \Omega_{0}\right)_{n \in \mathbb{N}}$ converging in norm to $\chi$. Passing to a subsequence, we can also assume that $\pi_{z}\left(\underline{A}_{n}\right) \Omega_{z} \rightarrow \chi(z)$ in norm for almost every $z \in \mathcal{Z}$. Hence, using the dominated convergence theorem and (i), we see that

$$
\lim _{n \rightarrow+\infty} \int_{\mathcal{Z}}^{\Gamma} d \nu(z) U_{\mu^{-1} . z}(\mu) \pi_{\mu^{-1} . z}\left(\underline{A}_{n}\right) \Omega_{\mu^{-1} . z}=\int_{\mathcal{Z}}^{\Gamma} d \nu(z) U_{\mu^{-1} . z}(\mu) \chi\left(\mu^{-1} . z\right),
$$

which gives (v).

Thus dilations act between the fibers of the direct integral decomposition by unitaries $U_{z}(\mu)$, which depend on the fiber. They fulfill the cocycle-type composition rule $U_{\mu . z}\left(\mu^{\prime}\right) U_{z}(\mu)=$ $U_{z}\left(\mu^{\prime} \mu\right)$ that one would naively expect; cf. also the theory of equivariant disintegrations for separable $C^{*}$ algebras Tak02, Ch. X §3].

We shall now further restrict to the situation of a factorizing scaling limit, as in Def. 4.3, in which the fiber spaces $\mathcal{H}_{z}$ are all identified with a unique space $\mathcal{H}_{\mathrm{u}}$. By this identification, we can regard the unitaries $U_{z}(\mu)$ as endomorphisms $\hat{U}_{z}(\mu)$ of $\mathcal{H}_{\mathrm{u}}$. Our result for these endomorphisms is as follows. 
Proposition 5.2. Let $\underline{\omega}_{0}$ be an invariant limit state. Suppose that the scaling limit of $\underline{\mathfrak{A}}$ is factorizing, and let $V=\int d \nu(z) V_{z}$ be the unitary of Def. 4.3. Then, the unitary operators

$$
\hat{U}_{z}(\mu): \mathcal{H}_{\mathrm{u}} \rightarrow \mathcal{H}_{\mathrm{u}}, \quad \hat{U}_{z}(\mu)=V_{\mu . z} U_{z}(\mu) V_{z}^{*}
$$

fulfill for any $z \in \mathcal{Z}, \mu, \mu^{\prime} \in \mathbb{R}_{+}$the relations

$$
\hat{U}_{z}(1)=\mathbf{1}, \quad \hat{U}_{z}(\mu)^{*}=\hat{U}_{\mu . z}\left(\mu^{-1}\right), \quad \hat{U}_{\mu . z}\left(\mu^{\prime}\right) \hat{U}_{z}(\mu)=\hat{U}_{z}\left(\mu^{\prime} \mu\right) .
$$

If $\mathcal{H}_{0}$ is uniformly separable, one has

$$
V U_{0}(\mu) V^{*}=\left(U_{\mathfrak{Z}}(\mu) \otimes \mathbf{1}\right) \int_{\mathcal{Z}}^{\oplus} d \nu(z) \hat{U}_{z}(\mu)
$$

and for every $\mu>0$, there is a null set $\mathcal{N} \subset \mathcal{Z}$ such that for any $(x, \Lambda) \in \mathcal{P}_{+}^{\uparrow}$ and any $z \in \mathcal{Z} \backslash \mathcal{N}$

$$
\hat{U}_{z}(\mu) U_{\mathrm{u}}(x, \Lambda)=U_{\mathrm{u}}(\mu x, \Lambda) \hat{U}_{z}(\mu) .
$$

Proof. It is clear that $\hat{U}_{z}(\mu)$, defined as above, are unitary, and their composition relations follow from Prop. 5.1 (iii), Now let $\mathcal{H}_{0}$ be uniformly separable. Then, together with $V$, also $V^{*}$ is decomposable. By a short computation, one finds for any $\chi \in L^{2}\left(\mathcal{Z}, \nu, \mathcal{H}_{\mathrm{u}}\right)$ :

$$
V U_{0}(\mu) V^{*} \chi=\int_{\mathcal{Z}}^{\oplus} d \nu(z) V_{z} U_{\mu^{-1} . z}(\mu) V_{\mu^{-1} . z}^{*} \chi\left(\mu^{-1} . z\right) .
$$

Now, following Prop. [5.1) (ii), the operator $U_{\mathfrak{Z}}(\mu) \otimes \mathbf{1}$ acts on vectors $\chi^{\prime}$ via

$$
\left(U_{\mathfrak{Z}}(\mu) \otimes \mathbf{1}\right) \chi^{\prime}=\int_{\mathcal{Z}}^{\oplus} d \nu(z) \chi^{\prime}\left(\mu^{-1} . z\right) .
$$

Together with Eq. (5.5), this entails

$$
V U_{0}(\mu) V^{*} \chi=\left(U_{\mathfrak{Z}}(\mu) \otimes \mathbf{1}\right) \int_{\mathcal{Z}}^{\oplus} d \nu(z) V_{\mu . z} U_{z}(\mu) V_{z}^{*} \chi(z),
$$

of which the second assertion follows. Further, one computes from $V U_{0}(x, \Lambda) V^{*}=\mathbf{1} \otimes U_{\mathrm{u}}(x, \Lambda)$ and from Eq. (5.7) that

$$
\int_{\mathcal{Z}}^{\oplus} d \nu(z) \hat{U}_{z}(\mu) U_{\mathbf{u}}(x, \Lambda)=V U_{0}(\mu, x, \Lambda) V^{*}=\int_{\mathcal{Z}}^{\oplus} d \nu(z) U_{\mathbf{u}}(\mu x, \Lambda) \hat{U}_{z}(\mu) .
$$

Uniform separability implies that the integrands agree except on a null set. This null set may depend on $x, \Lambda$. However, we can choose it uniformly on a countable dense set of the group, and hence, by continuity, uniformly for all group elements.

This shows that the dilation symmetries factorize into a central part, $U_{\mathfrak{Z}}(\mu) \otimes 1$, which "mixes" the fibers of the direct integral, and a decomposable part, $\int_{\mathcal{Z}}^{\oplus} d \nu(z) \hat{U}_{z}(\mu)$. The unitaries $\hat{U}_{z}(\mu)$ will generally depend on $z$; and like the $U_{z}(\mu)$ before, they do not necessarily fulfill a group relation, but a cocycle equation

$$
\hat{U}_{\mu . z}\left(\mu^{\prime}\right) \hat{U}_{z}(\mu)=\hat{U}_{z}\left(\mu^{\prime} \mu\right)
$$


as shown above, where $\mu . z$ can in general not be replaced with $z$. However, using the commutation relations with the other parts of the symmetry group, one sees that $\hat{U}_{z}\left(\mu^{\prime}\right) \hat{U}_{z}(\mu) \hat{U}_{z}\left(\mu^{\prime} \mu\right)^{*}$ is (a. e.) an inner symmetry of the theory $\mathfrak{A}_{\mathrm{u}}$. On the other hand, this representation property "up to an inner symmetry" cannot be avoided if such symmetries exist in the theory at all; for they might be multiplied to $V_{z}$ in a virtually arbitrary fashion at any point $z$. In this respect, we encounter a similar situation with respect to dilation symmetries as Buchholz and Verch BV95]. In the present context, however, it seems more transparent how this cocycle arises.

Under somewhat stricter assumptions, we can prove a stronger result that avoids the ambiguities discussed above. Let us consider the case of a convergent scaling limit, per Def. 4.4. In this case, we shall see that the $U_{z}(\mu)$ can actually be chosen independent of $z$, and yield a group representation in the usual sense.

Proposition 5.3. Let $\mathfrak{A}$ have a convergent scaling limit, and let $\underline{\omega}_{\mathrm{u}}$ be a multiplicative limit state with separable representation space $\mathcal{H}_{\mathrm{u}}$. Then the Poincaré group representation $U_{\mathrm{u}}$ on $\mathcal{H}_{\mathrm{u}}$ extends to a representation of the extended symmetry group $\underline{\mathcal{G}}$. For any invariant limit state $\underline{\omega}_{0}$ with associated representation $U_{0}$ of $\underline{\mathcal{G}}$, one has

$$
V U_{0}(\mu) V^{*}=\left(U_{0}(\mu)\left\lceil\mathcal{H}_{\mathfrak{Z}}\right) \otimes U_{\mathrm{u}}(\mu),\right.
$$

where $V$ is the unitary introduced in Proposition 4.10.

Proof. With $\underline{\omega}_{\mathrm{u}}$, also every $\underline{\omega}_{\mathrm{u}} \circ \underline{\delta}_{\mu}$ is a scaling limit state. Thanks to the invariance of $\underline{\mathfrak{A}}_{\text {conv }}$ under dilations, we thus have for each $\underline{A} \in \underline{\mathfrak{A}}_{\mathrm{conv}}$,

$$
\left\|\pi_{\mathrm{u}}\left(\underline{\delta}_{\mu}(\underline{A})\right) \Omega_{\mathrm{u}}\right\|^{2}=\underline{\omega}_{\mathrm{u}} \circ \underline{\delta}_{\mu}\left(\underline{A}^{*} \underline{A}\right)=\underline{\omega}_{\mathrm{u}}\left(\underline{A}^{*} \underline{A}\right)=\left\|\pi_{\mathrm{u}}(\underline{A}) \Omega_{\mathrm{u}}\right\|^{2} .
$$

This yields the existence of a unitary strongly continuous representation $\mu \mapsto U_{\mathrm{u}}(\mu)$ on $\mathcal{H}_{\mathrm{u}}$ such that

$$
U_{\mathrm{u}}(\mu) \pi_{\mathrm{u}}(\underline{A}) \Omega_{\mathrm{u}}=\pi_{\mathrm{u}}\left(\underline{\delta}_{\mu}(\underline{A})\right) \Omega_{\mathrm{u}}, \quad \underline{A} \in \underline{\mathfrak{A}}_{\text {conv }} .
$$

That also implies

$$
U_{\mathrm{u}}(\mu) U_{\mathrm{u}}(x, \Lambda) \pi_{\mathrm{u}}(\underline{A}) \Omega_{\mathrm{u}}=\pi_{\mathrm{u}}\left(\underline{\alpha}_{\mu, x, \Lambda}(\underline{A})\right) \Omega_{\mathrm{u}}, \quad \underline{A} \in \underline{\mathfrak{A}}_{\mathrm{conv}},
$$

which shows that $(\mu, \Lambda, x) \mapsto U_{\mathrm{u}}(\mu) U_{\mathrm{u}}(\Lambda, x)$ is a unitary representation of $\underline{\mathcal{G}}$ on $\mathcal{H}_{\mathrm{u}}$, extending the representation of the Poincaré group.

Now if $V: \mathcal{H}_{0} \rightarrow \mathcal{H}_{\mathfrak{Z}} \otimes \mathcal{H}_{\mathrm{u}}$ is the unitary of Prop. 4.10, a calculation shows that

$$
V U_{0}(\mu) V^{*}\left(\pi_{0}(\underline{C}) \Omega_{0} \otimes \pi_{\mathrm{u}}(\underline{A}) \Omega_{\mathrm{u}}\right)=\pi_{0}\left(\underline{\delta}_{\mu}(\underline{C})\right) \Omega_{0} \otimes \pi_{\mathrm{u}}\left(\underline{\delta}_{\mu}(\underline{A})\right) \Omega_{\mathrm{u}}, \quad \underline{C} \in \mathfrak{Z}(\underline{\mathfrak{A}}), \underline{A} \in \underline{\mathfrak{A}}_{\mathrm{conv}},
$$

which entails that $V U_{0}(\mu) V^{*}=\left(U_{0}(\mu)\left\lceil\mathcal{H}_{\mathfrak{Z}}\right) \otimes U_{\mathbf{u}}(\mu)\right.$.

Thus, the limit theory is "dilation covariant" in the usual sense, with a unitary acting on $\mathcal{H}_{\mathrm{u}}$. Considering the unitaries $\mathbf{1} \otimes U_{\mathrm{u}}(g)$, we actually get a unitary representation in any limit theory, even corresponding to multiplicative states. Only for compatibility with the scaling limit representation $\pi_{0}$ it is necessary to consider invariant means, and to take $U_{0}(\mu)\left\lceil\mathcal{H}_{\mathfrak{Z}}\right.$ into account. 


\section{Phase space properties}

In this section, we wish to investigate how the notion of phase space conditions, specifically the (quite weak) Haag-Swieca compactness condition [HS65], fits into our context, and how it transfers to the limit theory. An important aspect here is that Haag-Swieca compactness of a quantum field theory guarantees that the corresponding Hilbert space is separable; this property transfers to multiplicative limit states in certain circumstances Buc96a. We shall give a strengthened version of the compactness condition that guarantees our general limit spaces to be uniformly separable, a property that turned out to be valuable in the previous sections.

We need some extra structures to that end. First, we consider "properly rescaled" vectorvalued functions $\chi: \mathbb{R}_{+} \rightarrow \mathcal{H}$. Specifically, for $\underline{A} \in \underline{\mathfrak{A}}$, let $\underline{A} \Omega$ denote the function $\lambda \mapsto \underline{A}_{\lambda} \Omega$. We set

$$
\underline{\mathcal{H}}=\operatorname{clos}\{\underline{A} \Omega \mid \underline{A} \in \underline{\mathfrak{A}}\},
$$

where the closure is taken in the supremum norm $\|\underline{\chi}\|=\sup _{\lambda}\left\|\underline{\chi}_{\lambda}\right\|$. Then $\underline{\mathcal{H}}$ is a Banach space, in fact a Banach module over $\mathfrak{Z}(\underline{\mathfrak{A}})$ in a natural way. Given a limit state, we transfer the limit representation $\pi_{0}$ to vector-valued functions. To that end, consider the space $\mathcal{C}(\Gamma)$ of $\Gamma$-continuous vector fields, as defined in the Appendix. We define $\eta_{0}: \underline{\mathcal{H}} \rightarrow \mathcal{C}(\Gamma)$ on a dense set by

$$
\eta_{0}(\underline{A} \Omega):=\pi_{0}(\underline{A}) \Omega_{0} .
$$

This is well-defined, since one computes

$$
\begin{aligned}
\left\|\pi_{0}(\underline{A}) \Omega_{0}\right\|_{\infty}=\sup _{z \in \mathcal{Z}}\left\|\pi_{z}(\underline{A}) \Omega_{z}\right\|=\left(\sup _{z \in \mathcal{Z}} \underline{\omega}_{z}\left(\underline{A}^{*} \underline{A}\right)\right)^{1 / 2} & \\
& \leq\left\|\omega\left(\underline{A}^{*} \underline{A}\right)\right\|^{1 / 2}=\left(\sup _{\lambda>0}\left\|\underline{A}_{\lambda} \Omega\right\|^{2}\right)^{1 / 2}=\|\underline{A} \Omega\| .
\end{aligned}
$$

That also shows $\left\|\eta_{0}\right\| \leq 1$. Note that $\eta_{0}$ fulfills

$$
\eta_{0}(\underline{C \chi})=\pi_{0}(\underline{C}) \eta_{0}(\chi) \quad \text { for all } \underline{C} \in \mathfrak{Z}(\underline{\mathfrak{A}}), \chi \in \underline{\mathcal{H}},
$$

this easily being checked for $\chi=\underline{A} \Omega$. So $\eta_{0}$ preserves the module structure in this sense. Further, $\eta_{0}: \underline{\mathcal{H}} \rightarrow \mathcal{C}(\Gamma)$ clearly has dense range.

It is important in our context that $\underline{\mathcal{H}}$ is left invariant under multiplication with suitably rescaled functions of the Hamiltonian. More precisely, we denote these functions as $f(\underline{H})$ for $f \in \mathcal{S}\left(\mathbb{R}_{+}\right)$; they are defined as elements of $\underline{\mathfrak{B}}$ by $f(\underline{H})_{\lambda}=f(\lambda H)$, with norm $\|f(\underline{H})\| \leq\|f\|_{\infty}$. They act on $\underline{\mathcal{H}}$ by pointwise multiplication. The following lemma generalizes an observation in Buc96a].

Lemma 6.1. Let $f \in \mathcal{S}\left(\mathbb{R}_{+}\right)$. Then, for each $\underline{\chi} \in \underline{\mathcal{H}}$, we have $f(\underline{H}) \underline{\chi} \in \underline{\mathcal{H}}$. There exists a test function $g \in \mathcal{S}(\mathbb{R})$ such that for all $\underline{A} \in \underline{\mathfrak{A}}$,

$$
f(\underline{H}) \underline{A} \Omega=\underline{\alpha}_{g} \underline{A} \Omega:=\left(\int d t g(t) \underline{\alpha}_{t} \underline{A}\right) \Omega .
$$

Proof. We continue $f$ to a test function $\hat{f} \in \mathcal{S}(\mathbb{R})$, and choose $g$ as the Fourier transform of $\hat{f}$. One finds by spectral analysis of $H$ that for any $\underline{A} \in \underline{\mathfrak{A}}$,

$$
f(\lambda H) \underline{A}_{\lambda} \Omega=\int_{0}^{\infty} \hat{f}(\lambda E) d P(E) \underline{A}_{\lambda} \Omega=\int d t g(t) e^{\imath \lambda H t} \underline{A}_{\lambda} \Omega=\left(\underline{\alpha}_{g} \underline{A}\right)_{\lambda} \Omega .
$$


This shows that $f(\underline{H}) \underline{A} \Omega$ has the proposed form, and is an element of $\underline{\mathcal{H}}$. Since $\|f(\lambda H)\| \leq$ $\|f\|_{\infty}$ uniformly in $\lambda$, we may pass to limits in $\underline{A} \Omega$ and obtain that $f(\underline{H}) \underline{\chi} \in \underline{\mathcal{H}}$ for all $\chi \in \mathcal{H}$.

As a next step towards phase space conditions, let us explain a notion of compact maps adapted to our context. To that end, let $\mathcal{E}$ be a Banach space and $\mathcal{F}$ a Banach module over the commutative Banach algebra $\mathcal{R}$. We say that a linear map $\psi: \mathcal{E} \rightarrow \mathcal{F}$ is of uniform rank 1 if it is of the form $\psi=e(\cdot) f$ with $e: \mathcal{E} \rightarrow \mathcal{R}$ linear and continuous, and $f \in \mathcal{F}$. Sums of $n$ such terms are called of uniform rank $n$ We say that $\psi$ is uniformly compact if it is an infinite sum of terms of uniform $\operatorname{rank} 1, \psi=\sum_{j=0}^{\infty} e_{j}(\cdot) f_{j}$, where the sum converges in the Banach norm. For $\mathcal{R}=\mathbb{C}$, these definitions reduce to the usual notions of compact or finite-rank maps.

We are now in the position to consider Haag-Swieca compactness. We fix, once and for all, an element $\underline{C}^{<} \in \mathfrak{Z}(\underline{\mathfrak{A}})$ with $\left\|\underline{C}^{<}\right\| \leq 1, \underline{C}_{\lambda}^{<}=0$ for $\lambda>1$, and $\underline{C}_{\lambda}^{<}=1$ for $\lambda<1 / 2$. For a given $\beta>0$ and any bounded region $\mathcal{O}$, we consider the map

$$
\underline{\Theta}^{(\beta, \mathcal{O})}: \underline{\mathfrak{A}}(\mathcal{O}) \rightarrow \underline{\mathcal{H}}, \quad \underline{A} \mapsto e^{-\beta \underline{H}} \underline{C}^{<} \underline{A} \Omega .
$$

This is indeed well-defined due to Lemma 6.1. Our variant of the Haag-Swieca compactness condition, uniform at small scales, is then as follows.

Definition 6.2. A quantum field theory fulfills the uniform Haag-Swieca compactness condition if, for each bounded region $\mathcal{O}$, there is $\beta>0$ such that the map $\underline{\Theta}^{(\beta, \mathcal{O})}$ is uniformly compact.

We note that this property is independent of the choice of $\underline{C}^{<}$; the role of that factor is to ensure that we restrict our attention to the short-distance rather than the long-distance regime. We do not discuss relations of uniform Haag-Swieca compactness with other versions of phase space conditions here. Rather, we show in Sec. 7 that the condition is fulfilled in some simple models.

We now investigate how the compactness property transfers to the scaling limit. To that end, we consider the corresponding phase space map in the limit theory,

$$
\Theta_{0}^{(\beta, \mathcal{O})}: \mathfrak{A}_{0}(\mathcal{O}) \rightarrow \mathcal{H}_{0}, \quad A \mapsto e^{-\beta H_{0}} A \Omega_{0} .
$$

Its relation to $\underline{\Theta}^{(\beta, \mathcal{O})}$ is rather direct.

Proposition 6.3. For any fixed $\mathcal{O}$ and $\beta>0$, one has $\eta_{0} \circ \underline{\Theta}^{(\beta, \mathcal{O})}=\Theta_{0}^{(\beta, \mathcal{O})} \circ \pi_{0}$. If $\underline{\Theta}^{(\beta, \mathcal{O})}$ is uniformly compact, so is $\Theta_{0}^{(\beta, \mathcal{O})} \circ \pi_{0}$.

Proof. Given $\beta$, we choose a function $g_{\beta}$ relating to $f_{\beta}(E)=\exp (-\beta E)$ per Lemma 6.1. For any $\underline{A} \in \underline{\mathfrak{A}}(\mathcal{O})$, we compute

$$
\eta_{0} \underline{\Theta}^{(\beta, \mathcal{O})}(\underline{A})=\eta_{0}\left(\underline{C}^{<} \underline{\alpha}_{g_{\beta}} \underline{A} \Omega\right)=\pi_{0}\left(\underline{C}^{<}\right) \pi_{0}\left(\underline{\alpha}_{g_{\beta}} \underline{A}\right) \Omega_{0}=\alpha_{0, g_{\beta}} \pi_{0}(\underline{A}) \Omega_{0}=\Theta_{0}^{(\beta, \mathcal{O})} \pi_{0}(\underline{A}) .
$$

Thus $\eta_{0} \circ \underline{\Theta}^{(\beta, \mathcal{O})}=\Theta_{0}^{(\beta, \mathcal{O})} \circ \pi_{0}$ as proposed. Now let $\underline{\Theta}^{(\beta, \mathcal{O})}$ be uniformly compact, $\underline{\Theta}^{(\beta, \mathcal{O})}=$ $\sum_{j} e_{j}(\cdot) f_{j}$. Then $\eta_{0}$ can be exchanged with the infinite sum due to continuity, which yields

$$
\Theta_{0}^{(\beta, \mathcal{O})} \circ \pi_{0}=\sum_{j} \eta_{0}\left(e_{j}(\cdot) f_{j}\right)=\sum_{j}\left(\pi_{0} \circ e_{j}(\cdot)\right)\left(\eta_{0} f_{j}\right)
$$

\footnotetext{
${ }^{4}$ Note that the "uniform rank" is rather an upper estimate, in the sense that a map of uniform rank $n$ may at the same time be of uniform rank $n-1$.
} 
using Eq. (6.4). Thus $\Theta_{0}^{(\beta, \mathcal{O})} \circ \pi_{0}$ is uniformly compact.

The above results show in particular that img $\Theta_{0}^{(\beta, \mathcal{O})} \circ \pi_{0} \subset \mathcal{C}(\Gamma)$. Since we can write

$$
\Theta_{0}^{(\beta, \mathcal{O})} \circ \pi_{0}(\underline{A})=\int d \nu(z) \Theta_{z}^{(\beta, \mathcal{O})} \circ \pi_{z}(\underline{A})
$$

with the obvious definition of $\Theta_{z}^{(\beta, \mathcal{O})}$, the above proposition establishes a rather strong form of compactness in the limit theory, uniform in $z$; note that the sum in Eq. (6.9) converges with respect to the supremum norm.

We now come to the main result of the section, showing that compactness in the above form implies uniform separability of the limit Hilbert space.

Theorem 6.4. Suppose that the theory $\mathfrak{A}$ fulfils uniform Haag-Swieca compactness. Then, for any limit state $\underline{\omega}_{0}$, the representation space $\mathcal{H}_{0}$ is uniformly separable, where the fundamental sequence can be chosen from $\mathcal{C}(\Gamma)$.

Proof. We choose a sequence of regions $\mathcal{O}_{k}$ such that $\mathcal{O}_{k} \nearrow \mathbb{R}^{s+1}$, and a sequence $\left(\beta_{k}\right)_{k \in \mathbb{N}}$ in $\mathbb{R}_{+}$such that all $\underline{\Theta}^{\left(\beta_{k}, \mathcal{O}_{k}\right)}$ are uniformly compact. By Prop. 6.3 above, also $\Theta_{0}^{\left(\beta_{k}, \mathcal{O}_{k}\right)} \circ \pi_{0}$ are uniformly compact. Explicitly, choose $e_{j}^{(k)}: \underline{\mathfrak{A}}\left(\mathcal{O}_{k}\right) \rightarrow \mathcal{C}(\mathcal{Z})$ and $f_{j}^{(k)} \in \mathcal{C}(\Gamma)$ such that

$$
\Theta_{0}^{\left(\beta_{k}, \mathcal{O}_{k}\right)} \circ \pi_{0}=\sum_{j} e_{j}^{(k)}(\cdot) f_{j}^{(k)} .
$$

We will construct a fundamental sequence using the $f_{j}^{(k)}$. To that end, let $\underline{A} \in \underline{\mathfrak{A}}(\mathcal{O})$ for some $\mathcal{O}$. For $k$ large enough, we know that

$$
e^{-\beta H_{0}} \pi_{0}(\underline{A}) \Omega_{0}=\Theta_{0}^{\left(\beta_{k}, \mathcal{O}_{k}\right)}\left(\pi_{0}(\underline{A})\right)=\sum_{j} e_{j}^{(k)}(\underline{A}) f_{j}^{(k)} .
$$

The sum converges in the supremum norm, i.e., uniformly at all points $z$. Let us choose a fixed $z$. Then, it is clear that

$$
e^{-H_{z}^{2}} \pi_{z}(\underline{A}) \Omega_{z}=\sum_{j}\left(e_{j}^{(k)}(\underline{A})\right)(z) e^{-H_{z}^{2}+\beta_{k} H_{z}} f_{j}^{(k)}(z),
$$

noting that $\exp \left(-H_{z}^{2}+\beta_{k} H_{z}\right)$ is a bounded operator. Observe that $\left(e_{j}^{(k)}(\underline{A})\right)(z)$ are merely numerical factors. Since $\underline{A}$ and $\mathcal{O}$ were arbitrary, and $\cup_{\mathcal{O}} \pi_{z}(\underline{\mathfrak{A}}(\mathcal{O})) \Omega_{z}$ is dense in $\mathcal{H}_{z}$, this means

$$
e^{-H_{z}^{2}} \mathcal{H}_{z} \subset \operatorname{clos} \operatorname{span}\left\{e^{-H_{z}^{2}+\beta_{k} H_{z}} f_{j}^{(k)}(z) \mid j, k \in \mathbb{N}\right\} .
$$

Now $\exp \left(-H_{z}^{2}\right)$ is a selfadjoint operator with trivial kernel, thus its image is dense. Hence the $\exp \left(-H_{z}^{2}+\beta_{k} H_{z}\right) f_{j}^{(k)}(z)$ are total in $\mathcal{H}_{z}$. This holds for all $z$, thus $\left\{\exp \left(-H_{0}^{2}+\beta_{k} H_{0}\right) f_{j}^{(k)} \mid j, k \in\right.$ $\mathbb{N}\}$ is a fundamental sequence. Applying Lemma 6.1 to $f(E)=\exp \left(-E^{2}+\beta_{k} E\right)$, we find that the elements of the fundamental sequence lie in $\mathcal{C}(\Gamma)$. 


\section{Examples}

We are now going to investigate the structures discussed in simple models. Particularly, we wish to show that our conditions on "convergent scaling limits" (Def. 4.4) and "uniform HaagSwieca compactness" (Def. 6.2) can be fulfilled at least in simple situations. To that end, we first consider the situation where the theory $\mathfrak{A}$ "at finite scales" is equipped with a dilation symmetry. Then, we investigate the real scalar free field as a concrete example.

\subsection{Dilation covariant theories}

We now consider the case where the net $\mathfrak{A}$, which our investigation starts from, is already dilation covariant. One expects that the scaling limit construction reproduces the theory $\mathfrak{A}$ in this case, and that the dilation symmetry obtained from the scaling algebra coincides with the original one. We shall show that this is indeed the case under a mild phase space condition, and also that this implies the stronger phase space condition in Def. 6.2. This extends a discussion in [BV95, Sec. 5].

Technically, we will assume in the following that $\mathfrak{A}$ is a local net in the vacuum sector with symmetry group $\mathcal{G}$, which is generated by the Poincaré group and the dilation group. We shall denote the corresponding unitaries as $U(\mu, x, \Lambda)=U(\mu) U(x, \Lambda)$. The mild phase space condition referred to is the Haag-Swieca compactness condition for the original theory: We assume that for each bounded region $\mathcal{O}$ in Minkowski space, there exists $\beta>0$ such that the map $\Theta^{(\beta, \mathcal{O})}: \mathfrak{A}(\mathcal{O}) \rightarrow \mathcal{H}, A \mapsto \exp (-\beta H) A \Omega$ is compact. (This is equivalent to a formulation where the factor $\exp (-\beta H)$ is replaced with a sharp energy cutoff, as used in [HS65].)

Theorem 7.1. Let $\mathfrak{A}$ be a dilation covariant net in the vacuum sector which satisfies the Haag-Swieca compactness condition. Then $\mathfrak{A}$ has a convergent scaling limit.

Proof. We introduce the $\mathrm{C}^{*}$-subalgebra $\hat{\mathfrak{A}}(\mathcal{O}) \subset \mathfrak{A}(\mathcal{O})$ of those elements $A \in \mathfrak{A}(\mathcal{O})$ for which $g \mapsto \alpha_{g}(A)$ is norm continuous. Since the symmetries are implemented by continuous unitary groups, $\hat{\mathfrak{A}}(\mathcal{O})$ is strongly dense in $\mathfrak{A}(\mathcal{O})$. We then define a $\mathrm{C}^{*}$-subalgebra of the scaling algebra $\underline{\mathfrak{A}}(\mathcal{O})$,

$$
\underline{\mathfrak{A}}_{\text {conv }}(\mathcal{O}):=\left\{\lambda \mapsto U(\lambda) A U(\lambda)^{*} \mid A \in \hat{\mathfrak{A}}(\mathcal{O})\right\},
$$

and the $\underline{\alpha}$-invariant algebra $\underline{\mathfrak{A}}_{\text {conv }} \subset \underline{\mathfrak{A}}$ is defined as the $\mathrm{C}^{*}$-inductive limit of the $\underline{\mathfrak{A}}_{\text {conv }}(\mathcal{O})$.

It is evident that condition (i) in Def. 4.4 is fulfilled by $\underline{\mathfrak{A}}_{\text {conv }}$, as the functions $\lambda \mapsto \omega\left(\underline{A}_{\lambda}\right)$ are constant in the present case. Now let $\underline{\omega}_{0}$ be a multiplicative limit state. With similar argument.5 as in [BV95, Prop. 5.1], using the Haag-Swieca compactness condition, we can construct a net isomorphism $\phi$ from $\mathfrak{A}_{0}$ to $\mathfrak{A}$, which has the property that if $\underline{A}_{\lambda}=U(\lambda) A U(\lambda)^{*}$ with $A \in \hat{\mathfrak{A}}(\mathcal{O})$, then $\phi\left(\pi_{0}(\underline{A})\right)=A$. From this, and from the strong density of $\hat{\mathfrak{A}}(\mathcal{O})$ in $\mathfrak{A}(\mathcal{O})$, it follows that $\pi_{0}\left(\underline{\mathfrak{A}}_{\text {conv }}(\mathcal{O})\right)$ is strongly dense in $\mathfrak{A}_{0}(\mathcal{O})$. Thus condition (ii) in Def. 4.4 is satisfied as well.

Since the isomorphism $\phi$ above can be shown to intertwine the respective vacuum states, it is actually the adjoint action of a unitary $W: \mathcal{H}_{0} \rightarrow \mathcal{H}$. We remark that $\mathcal{H}$, and then also $\mathcal{H}_{0}$, is separable due to the Haag-Swieca compactness condition. Then as a consequence of Prop. 5.3, $\mathfrak{A}$ has a factorizing scaling limit and the representation of the symmetry group

\footnotetext{
${ }^{5}$ Since in contrast to BV95, we here take the $\mathfrak{A}_{0}(\mathcal{O})$ to be $\mathrm{W}^{*}$ algebras, we need to amend the argument in step (d) of [BV95. Prop. 5.1] slightly: We first construct the isomorphism $\phi$ on the $\mathrm{C}^{*}$ algebra $\pi_{0}(\underline{\mathfrak{A}}(\mathcal{O}))$, and then continue it to the weak closure; cf. [KR97, Lemma 10.1.10].
} 
$\mathcal{G}_{0}$ factorizes too. It is also clear from the proof above and from that of Thm. 4.9, that $\mathfrak{A}_{\mathrm{u}}$ is unitarily equivalent to $\mathfrak{A}$ through the operator $W$, taken here for $\pi_{\mathrm{u}}$ in place of $\pi_{0}$. Furthermore, this $W$ also intertwines the dilations in the scaling limit with those of the underlying theory.

Corollary 7.2. Under the hypothesis of Thm. 7.1, there holds

$$
W U_{\mathrm{u}}(\mu) W^{*}=U(\mu) .
$$

Proof. It is sufficient to verify the relation on vectors of the form $A \Omega$ with $A \in \hat{\mathfrak{A}}(\mathcal{O})$. For such vectors it follows by noting that $\underline{A}_{\lambda}=U(\lambda) A U(\lambda)^{*}$ is an element of $\underline{\mathfrak{A}}_{\text {conv }}(\mathcal{O})$, and that $\underline{\delta}_{\mu}(\underline{A})_{\lambda}=U(\lambda) \alpha_{\mu}(A) U(\lambda)^{*}$ with $\alpha_{\mu}(A) \in \hat{\mathfrak{A}}(\mu \mathcal{O})$.

For showing the consistency of our definitions, we now prove that the Haag-Swieca compactness condition at finite scales, together with dilation covariance, implies our uniform compactness condition of Def. 6.2.

Proposition 7.3. If the dilation covariant local net $\mathfrak{A}$ fulfills the Haag-Swieca compactness condition, then it also fulfills uniform Haag-Swieca compactness.

Proof. Let $\mathcal{O}$ be fixed, and let $\beta>0$ such that $\Theta^{(\beta, \mathcal{O})}$ is compact;

$$
\Theta^{(\beta, \mathcal{O})}=\sum_{j=1}^{\infty} e_{j}(\cdot) f_{j} \quad \text { with } e_{j} \in \mathfrak{A}(\mathcal{O})^{*}, f_{j} \in \mathcal{H} .
$$

Taking the normal part, we can in fact arrange that $e_{j} \in \mathfrak{A}(\mathcal{O})_{*}$. (See [BDF87, Lemma 2.2] for a similar argument.) Now define $\underline{e}_{j}: \underline{\mathfrak{A}}(\mathcal{O}) \rightarrow \mathfrak{Z}(\underline{\mathfrak{A}})$ by

$$
\underline{e}_{j}(\underline{A})_{\lambda}=e_{j}\left(U(\lambda)^{*} \underline{C}_{\lambda}^{<} \underline{A}_{\lambda} U(\lambda)\right) .
$$

That the image is indeed in $\mathfrak{Z}(\underline{\mathfrak{A}})$, i.e., continuous under $\underline{\delta}_{\mu}$, is seen as follows. We compute for $\lambda, \mu>0$,

$$
\begin{aligned}
\left|\underline{e}_{j}(\underline{A})_{\lambda \mu}-\underline{e}_{j}(\underline{A})_{\lambda}\right|= & \left|e_{j}\left(U(\lambda \mu)^{*}\left(\underline{C}^{<} \underline{A}\right)_{\lambda \mu} U(\lambda \mu)-U(\lambda)^{*}\left(\underline{C}^{<} \underline{A}\right)_{\lambda} U(\lambda)\right)\right| \\
& \leq\left\|e_{j}\right\|\left\|\underline{\delta}_{\mu}\left(\underline{C}^{<} \underline{A}\right)-\underline{C}^{<} \underline{A}\right\|+\left\|e_{j}\left(U(\mu)^{*} \cdot U(\mu)\right)-e_{j}\right\|\left\|\underline{C}^{<} \underline{A}\right\| .
\end{aligned}
$$

Now as $\mu \rightarrow 0$, the first summand vanishes due to norm continuity of $\underline{\delta}_{\mu}$ on $\underline{\mathfrak{A}}$, and the second due to strong continuity of $U(\mu)$; both limits are uniform in $\lambda$. Thus $\underline{\delta}_{\mu}$ acts continuously on $\underline{e}_{j}(\underline{A})$.

Further, we define $\underline{f}_{j} \in \underline{\mathcal{H}}$ by

$$
\underline{f}_{j \lambda}=U(\lambda) f_{j}
$$

This is indeed an element of $\underline{\mathcal{H}}$ : Namely, given $\epsilon>0$, choose $A \in \underline{\hat{\mathfrak{A}}}(\mathcal{O})$ for suitable $\mathcal{O}$ such that $\left\|A \Omega-f_{j}\right\|<\epsilon$; here $\hat{\mathfrak{A}}$ is as in the proof of Thm. 17.1. Then, $\underline{A}_{\lambda}=U(\lambda) A U(\lambda)^{*}$ defines an element of $\underline{\mathfrak{A}}$, and $\left\|\underline{A} \Omega-f_{j}\right\| \leq\left\|A \Omega-f_{j}\right\|<\epsilon$. Hence $\underline{f}_{j}$ is contained in the closure of $\underline{\mathfrak{A}} \Omega$. 
Now we are in the position to show that $\underline{\Theta}^{(\beta, \mathcal{O})}=\sum_{j} \underline{e}_{j} \underline{f}_{j}$. Let $J \in \mathbb{N}$ be fixed. It is straightforward to compute that for any $\underline{A} \in \underline{\mathfrak{A}}(\mathcal{O})$ and $\lambda>0$,

$$
\begin{aligned}
\left(\underline{\Theta}^{(\beta, \mathcal{O})}(\underline{A})-\sum_{j=1}^{J} \underline{e}_{j}(\underline{A}) \underline{f}_{j}\right)_{\lambda}=U(\lambda)\left(\Theta^{(\beta, \mathcal{O})}\left(B_{\lambda}\right)-\sum_{j=1}^{J} e_{j}\left(B_{\lambda}\right) f_{j}\right) \\
\quad \text { where } B_{\lambda}=U(\lambda)^{*} \underline{C}_{\lambda}^{<} \underline{A}_{\lambda} U(\lambda) .
\end{aligned}
$$

Note here that $B_{\lambda} \in \mathfrak{A}(\mathcal{O})$ for any $\lambda$. This entails

$$
\left\|\underline{\Theta}^{(\beta, \mathcal{O})}-\sum_{j=1}^{J} \underline{e}_{j}(\cdot) \underline{f}_{j}\right\| \leq\left\|\Theta^{(\beta, \mathcal{O})}-\sum_{j=1}^{J} e_{j}(\cdot) f_{j}\right\|\|\underline{A}\| .
$$

The right-hand sides vanishes as $J \rightarrow \infty$, as a consequence of the compactness condition at finite scales. This shows that $\underline{\Theta}^{(\beta, \mathcal{O})}$ is uniformly compact.

\subsection{The scaling limit of a free field}

We now show in a simple, concrete example from free field theory that the model has a convergent scaling limit in the sense of Def. 4.4. Specifically, we consider a real scalar free field of mass $m>0$, in $2+1$ or $3+1$ space-time dimensions. The algebraic scaling limit of this model is the massless real scalar field; this was as already discussed in [BV98], and in parts we rely on the arguments given there. However, we need to consider several aspects that were not handled in that work, in particular continuity aspects of Poincaré and dilation transformations. Also, as mentioned before, in contrast to BV98] we deal with weakly closed local algebras at fixed scales and in the limit theory.

We start by recalling, for convenience, the necessary notations and definitions from [BV98. We consider the Weyl algebra $\mathfrak{W}$ over $\mathcal{D}\left(\mathbb{R}^{s}\right), s=2,3$ :

$$
W(f) W(g)=e^{-\frac{2}{2} \sigma(f, g)} W(f+g), \quad \sigma(f, g)=\operatorname{Im} \int d^{s} x f(\mathbf{x}) g(\mathbf{x}),
$$

Then, we define a mass dependent automorphic action of $\mathcal{P}_{+}^{\uparrow}$ on $\mathfrak{W}$ by

$$
\alpha_{x, \Lambda}^{(m)}(W(f))=W\left(\tau_{x, \Lambda}^{(m)} f\right)
$$

where the action $\tau^{(m)}$ of $\mathcal{P}_{+}^{\uparrow}$ on $\mathcal{D}\left(\mathbb{R}^{s}\right)$ is defined by the following formulas. In those, we write $\tilde{f}(\mathbf{p})=(2 \pi)^{-s / 2} \int d \mathbf{x} f(\mathbf{x}) e^{-i \mathbf{x p}}$ for the Fourier transform of $f$, which we split into $\tilde{f}=\tilde{f}_{R}+\imath \tilde{f}_{I}$, where $f_{R}=\operatorname{Re} f$ and $f_{I}=\operatorname{Im} f ;$ also, $\omega_{m}(\mathbf{p}):=\sqrt{m^{2}+|\mathbf{p}|^{2}}$.

$$
\begin{aligned}
\left(\tau_{\mathbf{x}}^{(m)} f\right)(\mathbf{y}) & :=f(\mathbf{y}-\mathbf{x}) \\
\left(\tau_{t}^{(m)} f\right)_{R}(\mathbf{p}) & :=\cos \left(t \omega_{m}(\mathbf{p})\right) \tilde{f}_{R}(\mathbf{p})-\omega_{m}(\mathbf{p}) \sin \left(t \omega_{m}(\mathbf{p})\right) \tilde{f}_{I}(\mathbf{p}), \\
\left(\tau_{t}^{(m)} f\right)_{I}(\mathbf{p}) & :=\cos \left(t \omega_{m}(\mathbf{p})\right) \tilde{f}_{I}(\mathbf{p})+\omega_{m}(\mathbf{p})^{-1} \sin \left(t \omega_{m}(\mathbf{p})\right) \tilde{f}_{R}(\mathbf{p}), \\
\left(\tau_{\Lambda}^{(m)} f\right)_{R}(\mathbf{p}) & :=\varphi_{\Lambda}^{f}\left(\omega_{m}(\mathbf{p}), \mathbf{p}\right) \\
\left(\tau_{\Lambda}^{(m)} f\right)_{I}(\mathbf{p}) & :=\omega_{m}(\mathbf{p})^{-1} \psi_{\Lambda}^{f}\left(\omega_{m}(\mathbf{p}), \mathbf{p}\right) .
\end{aligned}
$$


Here the functions $\varphi_{\Lambda}^{f}, \psi_{\Lambda}^{f}: \mathbb{R}^{s+1} \rightarrow \mathbb{C}$ are defined by

$$
\begin{aligned}
& \varphi_{\Lambda}^{f}(p):=\frac{1}{2}\left(\tilde{f}_{R}\left(\boldsymbol{\Lambda}^{-1} \mathbf{p}\right)+\tilde{f}_{R}\left(\boldsymbol{\Lambda}^{T} \mathbf{p}\right)\right)+\frac{1}{2 \imath}\left(\left(\Lambda^{-1} p\right)_{0} \tilde{f}_{I}\left(\boldsymbol{\Lambda}^{-1} \mathbf{p}\right)-\left(\Lambda^{T} p\right)_{0} \tilde{f}_{I}\left(\boldsymbol{\Lambda}^{T} \mathbf{p}\right)\right), \\
& \psi_{\Lambda}^{f}(p):=-\frac{1}{2 \imath}\left(\tilde{f}_{R}\left(\boldsymbol{\Lambda}^{-1} \mathbf{p}\right)-\tilde{f}_{R}\left(\boldsymbol{\Lambda}^{T} \mathbf{p}\right)\right)+\frac{1}{2}\left(\left(\Lambda^{-1} p\right)_{0} \tilde{f}_{I}\left(\boldsymbol{\Lambda}^{-1} \mathbf{p}\right)+\left(\Lambda^{T} p\right)_{0} \tilde{f}_{I}\left(\boldsymbol{\Lambda}^{T} \mathbf{p}\right)\right),
\end{aligned}
$$

where we use the notation $\Lambda p=\left((\Lambda p)_{0}, \Lambda \mathbf{p}\right)$ for $\Lambda \in \mathcal{L}, p \in \mathbb{R}^{s+1}$. One verifies that all the above expressions are even in $\omega_{m}(\mathbf{p})$, which, due to the analytic properties of $\tilde{f}$, implies that $\tau_{x, \Lambda}^{(m)} \mathcal{D}\left(\mathbb{R}^{s}\right) \subset \mathcal{D}\left(\mathbb{R}^{s}\right)$. We also introduce the action $\sigma$ of dilations on $\mathfrak{W}$ by

$$
\sigma_{\lambda}(W(f))=W\left(\delta_{\lambda} f\right)
$$

with

$$
\left(\delta_{\lambda} f\right)(\mathbf{x}):=\lambda^{-(s+1) / 2}(\operatorname{Re} f)\left(\lambda^{-1} \mathbf{x}\right)+\imath \lambda^{-(s-1) / 2}(\operatorname{Im} f)\left(\lambda^{-1} \mathbf{x}\right) .
$$

It holds that $\alpha_{\lambda x, \Lambda}^{(m)} \circ \sigma_{\lambda}=\sigma_{\lambda} \circ \alpha_{x, \Lambda}^{(\lambda m)}$. Finally we define the vacuum state of mass $m \geq 0$ on $\mathfrak{W}$ as

$$
\omega^{(m)}(W(f))=e^{-\frac{1}{2}\|f\|_{m}^{2}}
$$

where

$$
\|f\|_{m}^{2}:=\frac{1}{2} \int_{\mathbb{R}^{s}} d \mathbf{p}\left|\omega_{m}(\mathbf{p})^{-1 / 2} \tilde{f}_{R}(\mathbf{p})+\imath \omega_{m}(\mathbf{p})^{1 / 2} \tilde{f}_{I}(\mathbf{p})\right|^{2} .
$$

There holds clearly $\omega^{(m)} \circ \alpha_{x, \Lambda}^{(m)}=\omega^{(m)}, \omega^{(m)} \circ \sigma_{\lambda}=\omega^{(\lambda m)}$.

Proceeding now along the lines of [BV98], we consider the GNS representation $\left(\pi^{(0)}, \mathcal{H}^{(0)}, \Omega^{(0)}\right)$ of $\mathfrak{W}$ induced by the massless vacuum state $\omega^{(0)}$. For each $m \geq 0$, we define a net $\mathcal{O} \mapsto$ $\mathfrak{A}^{(m)}(\mathcal{O})$ of von Neumann algebras on $\mathcal{H}^{(0)}$ as

$$
\mathfrak{A}^{(m)}\left(\Lambda \mathcal{O}_{B}+x\right):=\left\{\pi^{(0)}\left(\alpha_{x, \Lambda}^{(m)}(W(g))\right): \operatorname{supp} g \subset B\right\}^{\prime \prime},
$$

where $\mathcal{O}_{B}$ is any double cone with base the open ball $B$ in the time $t=0$ plane. For other open regions we can define the algebras by taking unions, but this will not be relevant for the following discussion. Due to the local normality of the different states $\omega^{(m)}, m \geq 0$, with respect to each other [EF74], these nets are isomorphic to the nets generated by the free scalar field of mass $m$ on the respective Fock spaces. From now on, we will identify elements of $\mathfrak{W}$ and of $\mathfrak{A}^{(m)}$, and therefore we will drop the indication of the representation $\pi^{(0)}$. We denote the (dilation and Poincaré covariant) scaling algebra associated to $\mathfrak{A}^{(m)}$ by $\underline{\mathfrak{A}}^{(m)}$. The next lemma generalizes the results of [BV98, Lemma 3.2] to the present situation.

Lemma 7.4. Let $a>1$ and $h^{D} \in \mathcal{D}((1 / a, a)), h^{P} \in \mathcal{D}\left(\mathcal{P}_{+}^{\uparrow}\right), f \in \mathcal{D}\left(\mathbb{R}^{s}\right)$, and consider the function $\underline{W}: \mathbb{R}_{+} \rightarrow \mathfrak{A}^{(m)}$ given by

$$
\underline{W}_{\lambda}:=\int_{\mathbb{R}_{+} \times \mathcal{P}_{+}^{\uparrow}} \frac{d \mu}{\mu} d x d \Lambda h^{D}(\mu) h^{P}(x, \Lambda) \alpha_{\mu \lambda x, \Lambda}^{(m)} \circ \sigma_{\mu \lambda}(W(f)),
$$

where $d \Lambda$ is the left-invariant Haar measure on the Lorentz group and the integral is to be understood in the weak sense. Then:

(i) there exists a double cone $\mathcal{O}$ such that $\underline{W} \in \underline{\mathfrak{A}}^{(m)}(\mathcal{O})$; 
(ii) there holds in the strong operator topology,

$$
\lim _{\lambda \rightarrow 0^{+}} \sigma_{\lambda}^{-1}\left(\underline{W}_{\lambda}\right)=\int_{\mathbb{R}_{+} \times \mathcal{P}_{+}^{\uparrow}} \frac{d \mu}{\mu} d x d \Lambda h^{D}(\mu) h^{P}(x, \Lambda) \alpha_{\mu x, \Lambda}^{(0)} \circ \sigma_{\mu}(W(f))=: W_{0} ;
$$

(iii) the span of the operators $W_{0}$ of the form above, with $\underline{W} \in \underline{\mathfrak{A}}^{(m)}(\mathcal{O})$ for fixed $\mathcal{O}$, is strongly dense in $\mathfrak{A}^{(m)}(\mathcal{O})$.

Proof. Since $\underline{W}$ is the convolution, with respect to the action $(\mu, x, \Lambda) \mapsto \underline{\alpha}_{\mu, x, \Lambda}^{(m)}$, of the function $h^{D} \otimes h^{P}$ with the bounded function $\lambda \mapsto \sigma_{\lambda}(W(f))$, and thanks to the support properties of $h^{D}, h^{P}$ and $f$, (i) follows.

In order to prove (ii), we start by observing that, for each vector $\chi \in \mathcal{H}^{(0)}$,

$$
\begin{aligned}
\left\|\left(\sigma_{\lambda}^{-1}\left(\underline{W}_{\lambda}\right)-W_{0}\right) \chi\right\| \leq \int_{\mathbb{R}_{+} \times \mathcal{P}_{+}^{\uparrow}} \frac{d \mu}{\mu} d x d \Lambda & \left|h^{D}(\mu) h^{P}(x, \Lambda)\right| \\
& \times\left\|\left(W\left(\delta_{\mu} \tau_{x, \Lambda}^{(\mu \lambda m)} f\right)-W\left(\delta_{\mu} \tau_{x, \Lambda}^{(0)} f\right)\right) \chi\right\| .
\end{aligned}
$$

Now $f \mapsto W(f)$ is known to be continuous with respect to $\|\cdot\|_{0}$ on the initial space and the strong operator topology on the target space [BR81, Prop. 5.2.4]. Since the norm $\|\cdot\|_{0}$ is $\delta_{\mu}$-invariant, it therefore suffices to show that for each fixed $(x, \Lambda) \in \mathcal{P}_{+}^{\uparrow}$,

$$
\lim _{m \rightarrow 0^{+}}\left\|\tau_{x, \Lambda}^{(m)} f-\tau_{x, \Lambda}^{(0)} f\right\|_{0}=0
$$

for (ii) then follows from the dominated convergence theorem. In order to show Eq. (7.20), we introduce the following family of functions $f^{(m)}(\mathbf{p})$ of two arguments:

$$
\begin{aligned}
\mathcal{F}=\left\{f:[0,1] \times \mathbb{R}^{s} \rightarrow \mathbb{C} \mid\right. & f^{(m)}(\cdot) \in \mathcal{D}\left(\mathbb{R}^{s}\right) \text { for each fixed } m \in[0,1] ; \\
& \lim _{m \rightarrow 0} \widetilde{f^{(m)}}(\mathbf{p})=\widetilde{f^{(0)}}(\mathbf{p}) \text { for each fixed } \mathbf{p} \in \mathbb{R}^{s} ; \\
& \left.\exists g \in \mathcal{S}\left(\mathbb{R}^{s}\right) \forall m \in[0,1] \forall \mathbf{p} \in \mathbb{R}^{s}:\left|\widetilde{f^{(m)}}(\mathbf{p})\right| \leq g(\mathbf{p})\right\} .
\end{aligned}
$$

It is clear that for $f \in \mathcal{F}$, one has $\left\|f^{(m)}-f^{(0)}\right\|_{0} \rightarrow 0$ as $m \rightarrow 0$ per dominated convergence. Also, each $f \in \mathcal{D}\left(\mathbb{R}^{s}\right)$, with trivial dependence on $m$, falls into $\mathcal{F}$. So it remains to show that the (naturally defined) action of $\tau_{x, \Lambda}^{(\cdot)}$ leaves $\mathcal{F}$ invariant, where it suffices to check this for a set of generating subgroups. Indeed, $\tau_{x, \Lambda}^{(\cdot)} \mathcal{F} \subset \mathcal{F}$ is clear for spatial translations and rotations. For time translations and boosts, it was already remarked that $\mathcal{D}\left(\mathbb{R}^{s}\right)$ is invariant under these at fixed $m$, and pointwise convergence as $m \rightarrow 0$ is clear. Further, from Eqs. (7.11) and (7.13), one sees that $\tilde{f}$ is modified by at most polynomially growing functions, uniform in $m \leq 1$, hence uniform $\mathcal{S}$-bounds hold for $\tau_{x, \Lambda}^{(m)} f^{(m)}$ as well. (Again, it enters here that all expressions are even in $\omega_{m}$, for which it is needed that $\tilde{f}$ is smooth.) This completes the proof of (ii).

Finally, (iii) follows from the observation that as $h_{D}$ and $h_{P}$ converge to delta functions, $W_{0}$ converges strongly to $W(f)$ thanks to the strong continuity of the function $(\mu, x, \Lambda) \mapsto$ $\alpha_{\mu x, \Lambda}^{(0)} \circ \sigma_{\mu}(W(f))$; and of course the span of the Weyl operators with supp $f \subset \subset \mathcal{O}$ is strongly dense in $\mathfrak{A}^{(m)}(\mathcal{O})$. 
Using the above lemma, we can prove the following.

Theorem 7.5. The theory of the massive real scalar free field in $s=2,3$ spatial dimensions has a convergent scaling limit.

Proof. Consider the $\mathrm{C}^{*}$-subalgebra $\underline{\mathfrak{A}}_{\mathrm{conv}}^{(m)}(\mathcal{O})$ of $\underline{\mathfrak{A}}^{(m)}(\mathcal{O})$ which is generated by the elements $\underline{W} \in \underline{\mathfrak{A}}^{(m)}(\mathcal{O})$ defined in the previous lemma, and let $\underline{\mathfrak{A}}_{\text {conv }}^{(m)}$ be the corresponding quasi-local algebra. Since $\underline{\alpha}_{\mu, x, \Lambda}^{(m)}(\underline{W})$ is again an element of the same form, just with shifted function $h^{D} \otimes h^{P}$, the algebra $\underline{\mathfrak{A}}_{\mathrm{conv}}^{(m)}$ is $\underline{\alpha}^{(m)}$ invariant. In order to verify that $\lambda \mapsto \omega^{(m)}\left(\underline{A}_{\lambda}\right)$ has a limit, as $\lambda \rightarrow 0$, for all $\underline{A} \in \underline{\mathfrak{A}}_{\mathrm{conv}}^{(m)}$, we start by observing that, thanks to Lemma 7.4 (ii) and to the fact that $\sigma_{\lambda}$ is unitarily implemented on $\mathcal{H}^{(0)}$, for each such $\underline{A}$ there exists $\lim _{\lambda \rightarrow 0^{+}} \sigma_{\lambda}^{-1}\left(\underline{A}_{\lambda}\right)=: A$ in the strong operator topology. Then if $\underline{A} \in \underline{\mathfrak{A}}_{\mathrm{conv}}^{(m)}(\mathcal{O})$ there holds the inequality

$$
\left|\omega^{(m)}\left(\underline{A}_{\lambda}\right)-\omega^{(0)}(A)\right| \leq \|\left(\omega^{(m)}-\omega^{(0)}\right)\left\lceil\mathfrak{A}^{(0)}(\lambda \mathcal{O})\|\| \underline{A} \|+\left|\omega^{(0)}\left(\sigma_{\lambda}^{-1}\left(\underline{A}_{\lambda}\right)\right)-\omega^{(0)}(A)\right| .\right.
$$

Together with the fact that $\lim _{\lambda \rightarrow 0^{+}} \|\left(\omega^{(m)}-\omega^{(0)}\right)\left\lceil\mathfrak{A}^{(0)}(\lambda \mathcal{O}) \|=0\right.$ as a consequence of the local normality of $\omega^{(m)}$ with respect to $\omega^{(0)}$, this implies that $\lim _{\lambda \rightarrow 0^{+}} \omega^{(m)}\left(\underline{A}_{\lambda}\right)=\omega^{(0)}(A)$ for all $\underline{A}$ in some local algebra $\underline{\mathfrak{A}}_{\mathrm{conv}}^{(m)}(\mathcal{O})$. This then extends to all of $\underline{\mathfrak{A}}_{\mathrm{conv}}^{(m)}$ by density.

It remains to show that for multiplicative limit states, $\pi_{0}\left(\underline{\mathfrak{A}}_{\text {conv }}^{(m)} \cap \underline{\mathfrak{A}}^{(m)}(\mathcal{O})\right)$ is weakly dense in $\mathfrak{A}_{0}^{(m)}(\mathcal{O})=\pi_{0}\left(\underline{\mathfrak{A}}^{(m)}(\mathcal{O})\right)^{\prime \prime}$ for any $\mathcal{O}$. To that end, we use similar methods as in Thm. 7.1. With $\mathcal{O}$ fixed and $U$ the ultrafilter that underlies the limit state, we define

$$
\phi: \pi_{0}\left(\underline{\mathfrak{A}}^{(m)}(\mathcal{O})\right) \rightarrow \mathfrak{A}^{(0)}(\mathcal{O}), \quad \pi_{0}(\underline{A}) \mapsto \lim _{U} \sigma_{\lambda}^{-1}\left(\underline{A}_{\lambda}\right),
$$

with the limit understood in the weak operator topology. Using methods as in [BV98, Sec. 3], one can show that $\phi$ is indeed a well-defined isometric * homomorphism, which further satisfies $\omega_{0}=\omega^{(0)} \circ \phi$ on the domain of $\phi$. Hence $\phi$ is given by the adjoint action of a partial isometry, and can be continued by weak closure to a ${ }^{*}$ homomorphism $\phi^{-}: \mathfrak{A}_{0}^{(m)}(\mathcal{O}) \rightarrow \mathfrak{A}^{(0)}(\mathcal{O})$. On the other hand, for $\underline{W} \in \underline{\mathfrak{A}}_{\text {conv }}^{(m)}(\mathcal{O})$ as in Lemma [7.4, one finds $\phi\left(\pi_{0}(\underline{W})\right)=W_{0}$ by (ii) of that lemma. However, the double commutant of those $W_{0}$ is all of $\mathfrak{A}^{(0)}(\mathcal{O})$, see (iii) of the same lemma. So $\phi^{-}$is in fact an isomorphism; and inverting $\phi^{-}$, one obtains the proposed density.

\subsection{Phase space conditions in the free field}

Our aim in this section is to prove the uniform compactness condition of Sec. 6 in the case of a real scalar free field, again of mass $m \geq 0$, in $3+1$ or higher dimensions. To that end, we will use a short-distance expansion of local operators, very similar to the method used in the Appendix of Bos05b], however in a refined formulation.

In this section, we will consider a fixed mass $m \geq 0$ throughout, and therefore we drop the label $(m)$ from the local algebras, the vacuum state, and the Hilbert space norm for simplicity. We rewrite the Weyl operators of Eq. (7.8) in terms of the familiar free field $\phi$ and its time derivative $\partial_{0} \phi$ in the time- 0 plane,

$$
W(f)=\exp \imath\left(\phi(\operatorname{Re} f)-\partial_{0} \phi(\operatorname{Im} f)\right), \quad f \in \mathcal{D}\left(\mathbb{R}^{s}\right) .
$$

Also, we need to introduce some multi-index notation. Given $n \in \mathbb{N}_{0}$, we consider multiindexes $\nu=\left(\nu_{1}, \ldots, \nu_{n}\right) \in\left(\{0,1\} \times \mathbb{N}_{0}^{s}\right)^{n}$; that is, each $\nu_{j}$ has the form $\nu_{j}=\left(\nu_{j 0}, \nu_{j 1}, \ldots, \nu_{j s}\right)$ 
with $\nu_{j 0} \in\{0,1\}, \nu_{j k} \in \mathbb{N}_{0}$ for $1 \leq k \leq s$. These indices will be used for labeling derivatives in configuration space, $\partial^{\nu_{j}}=\partial_{0}^{\nu_{j 0}} \ldots \partial_{s}^{\nu_{j s}}$. We denote

$$
\nu_{j} !=\prod_{k=0}^{s} \nu_{j k} !, \quad \nu !=\prod_{j=1}^{n} \nu_{j} !, \quad\left|\nu_{j}\right|=\sum_{k=0}^{s} \nu_{j k}, \quad|\nu|=\sum_{j=1}^{n}\left|\nu_{j}\right| .
$$

Now we can define the following local fields as quadratic forms on a dense domain.

$$
\phi_{n, \nu}=: \prod_{j=1}^{n} \partial^{\nu_{j}} \phi:(0) .
$$

These will form a basis in the space of local fields at $x=0$. Further, for given $r>0$, we choose a test function $h \in \mathcal{D}\left(\mathbb{R}^{s}\right)$ which is equal to 1 for $|\mathbf{x}| \leq r$; then we set $h_{\nu_{j}}(\mathbf{x})=\prod_{k=1}^{s} x_{k}^{\nu_{j k}} h(\mathbf{x})$. This is used to define the following functionals on $\mathfrak{A}\left(\mathcal{O}_{r}\right)$.

$$
\sigma_{n, \nu}(A)=\frac{\imath^{n}(-1)^{\sum_{j} \nu_{j 0}}}{n ! \nu !}\left(\Omega \mid\left[\partial_{0}^{\left(1-\nu_{10}\right)} \phi\left(h_{\nu_{1}}\right),\left[\ldots\left[\partial_{0}^{\left(1-\nu_{n 0}\right)} \phi\left(h_{\nu_{n}}\right), A\right] \ldots\right] \Omega\right) .\right.
$$

One sees that this definition is independent of the choice of $h$. We can therefore consistently consider $\sigma_{n, \nu}$ as a functional on $\cup_{\mathcal{O}} \mathfrak{A}(\mathcal{O})$, though its norm may increase as $\mathcal{O}$ grows large. The significance of these functionals becomes clear in the following lemma.

Lemma 7.6. We have for all Weyl operators $A=W(f)$ with $f \in \mathcal{D}\left(\mathbb{R}^{s}\right)$,

$$
A=\sum_{n=0}^{\infty} \sum_{\nu} \sigma_{n, \nu}(A) \phi_{n, \nu}
$$

in the sense of matrix elements between vectors of finite energy and finite particle number.

Proof. We indicate only briefly how this combinatorial formula can be obtained; see also [Bos00, Sec. 7.2] and [Bos05b, Appendix]. Using Wick ordering, we rewrite Eq. (7.24) for the Weyl operators as

$$
W(f)=e^{-\|f\|^{2} / 2}: \exp \imath\left(\phi(\operatorname{Re} f)-\partial_{0} \phi(\operatorname{Im} f)\right):=e^{-\|f\|^{2} / 2} \sum_{n=0}^{\infty} \frac{\imath^{n}}{n !}:\left(\phi(\operatorname{Re} f)-\partial_{0} \phi(\operatorname{Im} f)\right)^{n}: .
$$

Now, in each factor of the $n$-fold product $:(\ldots)^{n}$ :, we expand both $\operatorname{Re} f$ and $\operatorname{Im} f$ into a Taylor series in momentum space. Note that this is justified, since those functions have compact support in configuration space, since they are evaluated in scalar products with functions of compact support in momentum space, and since the sum over $n$ is finite in matrix elements. The Taylor expansion in momentum space corresponds to an expansion in derivatives of $\delta$ functions in configuration space, and this is what produces the fields $\phi_{n, \nu}$ localized at 0 . We then need to identify the remaining factors with $\sigma_{n, \nu}(A)$, which is done using the known commutation relations of $W(f)$ with $\phi$ and $\partial_{0} \phi$.

Our main task will now be to extend the above formula to more general states and more observables, by showing that the sum converges in a suitable norm. To that end, we need estimates of the fields and functionals involved. 
Lemma 7.7. Given $s \geq 2, m \geq 0$, and $r_{0}>0$, there exists a constant $c$ such that the following holds for any $n, \nu$.

$$
\begin{aligned}
\left\|e^{-\beta H} \phi_{n, \nu} \Omega\right\| & \leq c^{n}(n !)^{1 / 2} \nu !(\beta / 2)^{-|\nu|-n(s-1) / 2} & & \text { for any } \beta>0, \\
\left\|P(E) \phi_{n, \nu} P(E)\right\| & \leq c^{n} E^{|\nu|+n(s-1) / 2} & & \text { for any } E \geq 1, \text { provided } s \geq 3 \\
\| \sigma_{n, \nu}\left\lceil\mathfrak{A}\left(\mathcal{O}_{r}\right) \|\right. & \leq c^{n}(n !)^{-1 / 2}(\nu !)^{-1}(3 r)^{|\nu|+n(s-1) / 2} & & \text { for any } r \leq r_{0} .
\end{aligned}
$$

Proof. One has

$$
\left\|e^{-\beta H} \phi_{n, \nu} \Omega\right\|=\left\|\left(\prod_{j=1}^{n} a^{*}\left(e^{-\beta \omega} p^{\nu_{j}}\right)\right) \Omega\right\| \leq \sqrt{n !} \prod_{j=1}^{n}\left\|e^{-\beta \omega} p^{\nu_{j}}\right\| .
$$

For the single-particle vectors on the right-hand side, one uses scaling arguments to obtain the estimate

$$
\left\|e^{-\beta \omega} p^{\nu_{j}}\right\| \leq c_{1} \nu_{j} !(\beta / 2)^{-\left|\nu_{j}\right|-(s-1) / 2} \quad \text { for } \beta>0,
$$

where $c_{1}$ is a constant (depending on $s$ and $m$ ). This implies (国).-For (b) , we use energy bounds for creation operators $a^{*}(f)$, similar to [BP90, Sec. 3.3]. One finds for single particle space functions $f_{1}, \ldots, f_{k}$ in the domain of $\omega_{m}^{1 / 2}$,

$$
\left\|P(E) a^{*}\left(\omega_{m}^{1 / 2} f_{1}\right) \ldots a^{*}\left(\omega_{m}^{1 / 2} f_{k}\right)\right\| \leq E^{k / 2}\left\|Q(E) f_{1}\right\| \ldots\left\|Q(E) f_{k}\right\|,
$$

with $Q(E)$ being the energy projector for energy $E$ in single particle space. This leads us to

$$
\left\|P(E) \phi_{n, \nu} P(E)\right\| \leq 2^{n} E^{n / 2} \prod_{j=1}^{n}\left\|\omega_{m}^{-1 / 2} p^{\nu_{j}} \chi_{E}\right\|,
$$

where $p^{\nu_{j}}=\prod_{k=0}^{s} p_{k}^{\nu_{j k}}$, and $\chi_{E}$ is the characteristic function of $\omega_{m}(\mathbf{p}) \leq E$. For the singleparticle functions, one obtains

$$
\left\|\omega_{m}^{-1 / 2} p^{\nu_{j}} \chi_{E}\right\| \leq c_{2} E^{\left|\nu_{j}\right|+(s-2) / 2}
$$

with a constant $c_{2}$, which implies (b).

Now consider the functional $\sigma_{n, \nu}$. We choose a test function $h_{1} \in \mathcal{D}\left(\mathbb{R}_{+}\right)$such that $h_{1}(x) \leq$ 1 for all $x, h_{1}(x)=1$ on $[0,1]$, and $h_{1}(x)=0$ for $x \geq 2$. Then, $h_{r}(\mathbf{x})=h_{1}(|\mathbf{x}| / r)$ is a valid choice for the test function used in the definition of $\sigma_{n, \nu}\left\lceil\mathfrak{A}\left(\mathcal{O}_{r}\right)\right.$, see Eq. (7.27). Expressing the fields $\phi$ there in annihilation and creation operators, and writing each commutator as a sum of two terms, we obtain

$$
\| \sigma_{n, \nu}\left\lceil\mathfrak{A}\left(\mathcal{O}_{r}\right)\left\|\leq \frac{4^{n}}{\sqrt{n !} \nu !} \prod_{j=1}^{n}\right\| \omega_{m}^{\left(1-\nu_{j 0}\right)} h_{r, \nu_{j}} \| .\right.
$$

Again, we use scaling arguments for the single-particle space functions and obtain for $r \leq r_{0}$,

$$
\left\|\omega_{m}^{\left(1-\nu_{j 0}\right)} h_{r, \nu_{j}}\right\| \leq c_{3}(3 r)^{\left|\nu_{j}\right|+(s-1) / 2}
$$

with a constant $c_{3}$ that depends on $r_{0}$. This yields (ㄷ). 
We now define the "scale-covariant" objects that will allow us to expand the maps $\underline{\Theta}^{(\beta, \mathcal{O})}$ in a series. They are constructed of the fields $\phi_{n, \nu}$ and the functionals $\sigma_{n, \nu}$ by multiplication with appropriate powers of $\lambda$. We begin with the quantum fields.

Proposition 7.8. For any $n, \nu$ and $\beta>0$, the function

$$
\chi_{n, \nu, \beta}: \lambda \mapsto \lambda^{|\nu|+n(s-1) / 2} e^{-\beta \lambda H} \phi_{n, \nu} \Omega
$$

defines an element of $\underline{\mathcal{H}}$, with norm estimate $\left\|\chi_{n, \nu, \beta}\right\| \leq c^{n}(n !)^{1 / 2} \nu !(\beta / 2)^{-|\nu|-n(s-1) / 2}$.

Proof. We use techniques from BDM09, and adopt the notation introduced there. In particular, $\underline{R}$ denotes the function $\underline{R}_{\lambda}=(1+\lambda H)^{-1}$, and we write $\|\underline{A}\|^{(\ell)}=\sup _{\lambda}\left\|\underline{R}_{\lambda}^{\ell} \underline{A}_{\lambda} \underline{R}_{\lambda}^{\ell}\right\|$, where $\underline{A}_{\lambda}$ may be unbounded quadratic forms. Let $n, \nu$ be fixed in the following. We set

$$
\underline{\phi}_{\lambda}=\lambda^{|\nu|+n(s-1) / 2} \phi_{n, \nu} .
$$

From Lemma 7.7, one sees that $\left\|P(E / \lambda) \underline{\phi}_{\lambda} P(E / \lambda)\right\|$ is bounded uniformly in $\lambda$. Hence, applying [BDM09, Lemma 2.6], we obtain $\|\underline{\phi}\|^{(\ell)}<\infty$ for sufficiently large $\ell$. Also, the action $g \mapsto \underline{\alpha}_{g} \underline{\phi}$ of the symmetry group on $\underline{\phi}$ (which extends canonically from bounded operators to quadratic forms) is continuous in some $\|\cdot\|^{(\ell)}$ : This is clear for translations by the energy-damping factor; for dilations it is immediate from the definition; and for Lorentz transformations it holds since they act by a finite-dimensional matrix representation on $\phi_{n, \nu}$. Thus, $\underline{\phi}$ is an element of the space $\underline{\Phi}$ defined in [BDM09, Eq. (2.39)]. Moreover, each $\underline{\phi}_{\lambda}$ is clearly an element of the Fredenhagen-Hertel field content $\Phi_{\mathrm{FH}}$. Thus, BDM09, Thm. 3.8] provides us with a sequence $\left(\underline{A}_{n}\right)$ in $\mathfrak{A}(\mathcal{O})$, with $\mathcal{O}$ a fixed neighborhood of zero, such that $\left\|\underline{A}_{n}-\phi\right\|^{(\ell)} \rightarrow 0$ as $n \rightarrow \infty$. Now since $\left\|\exp (-\beta \underline{H}) \underline{R}^{-\ell}\right\|<\infty$, we obtain $\| \exp (-\beta \underline{H})\left(\underline{A}_{n}-\right.$ $\phi) \Omega \| \rightarrow 0$. Note here that $\exp (-\beta \underline{H}) \underline{A} \Omega \in \underline{\mathcal{H}}$ by Lemma 6.1. Hence $\exp (-\beta \underline{H}) \phi \Omega=\chi_{n, \nu, \beta}$ lies in $\underline{\mathcal{H}}$, since this space is closed in norm. The estimate for $\left\|\chi_{n, \nu, \beta}\right\|$ follows directly from Lemma 7.7](a).

Next, we rephrase the functionals $\sigma_{n, \nu}$ as maps from the scaling algebra $\underline{\mathfrak{A}}$ to its center.

Lemma 7.9. For any $n, \nu$, the definition

$$
\left(\underline{\sigma}_{n, \nu}(\underline{A})\right)_{\lambda}=\lambda^{-|\nu|-n(s-1) / 2} \sigma_{n, \nu}\left(\left(\underline{C}^{<} \underline{A}\right)_{\lambda}\right)
$$

yields a linear map $\underline{\sigma}_{n, \nu}: \cup_{\mathcal{O}} \underline{\mathfrak{A}}(\mathcal{O}) \rightarrow \mathfrak{Z}(\underline{\mathfrak{A}})$, with its norm bounded by

$$
\| \underline{\sigma}_{n, \nu}\left\lceil\underline{\mathfrak{A}}\left(\mathcal{O}_{r}\right) \| \leq c^{n}(\sqrt{n !} \nu !)^{-1}(3 r)^{|\nu|+n(s-1) / 2}\right.
$$

for $r \leq r_{0}$; here $r_{0}, c$ are the constants of Lemma 7.7.

Proof. The norm estimate is a consequence of Lemma 7.7(드), where one notes that $\left(\underline{C}^{<} \underline{A}\right)_{\lambda} \in$ $\mathfrak{A}\left(\mathcal{O}_{\lambda r}\right)$ for $\lambda \leq 1$, and $\left(\underline{C}^{<} \underline{A}\right)_{\lambda}=0$ for $\lambda>1$, so that these operators are always contained in $\mathfrak{A}\left(\mathcal{O}_{r_{0}}\right)$. It remains to show that $\underline{\sigma}_{n, \nu}(\underline{A}) \in \mathfrak{Z}(\underline{\mathfrak{A}})$, i.e., that $\mu \mapsto \underline{\delta}_{\mu}\left(\underline{\sigma}_{n, \nu}(\underline{A})\right)$ is continuous. But this follows from continuity of $\mu \mapsto \underline{\delta}_{\mu} \underline{A}$ and the definition of $\underline{\sigma}_{n, \nu}$.

We are now in the position to prove that $\sum_{n, \nu} \underline{\sigma}_{n, \nu} \chi_{n, \nu, \beta}$ is a norm convergent expansion of the map $\underline{\Theta}^{(\beta, \mathcal{O})}$. 
Theorem 7.10. Let $s \geq 3$. For each $r>0$, there exists $\beta>0$ such that

$$
\underline{\Theta}^{\left(\beta, \mathcal{O}_{r}\right)}=\sum_{n=0}^{\infty} \sum_{\nu} \underline{\sigma}_{n, \nu}(\cdot) \underline{\chi}_{n, \nu, \beta} .
$$

Proof. We will show below that the series in the statement converges in norm in the Banach space $\mathcal{B}\left(\underline{\mathfrak{A}}\left(\mathcal{O}_{r}\right), \underline{\mathcal{H}}\right)$, i.e. that

$$
\sum_{n=0}^{\infty} \sum_{\nu} \| \underline{\sigma}_{n, \nu}\left\lceil\underline{\mathfrak{A}}\left(\mathcal{O}_{r}\right)\|\| \underline{\chi}_{n, \nu, \beta} \|<\infty .\right.
$$

Once this has been established, the assertion of the theorem is obtained as follows. From Lemma 7.6, we know that

$$
\sum_{n=0}^{\infty} \sum_{\nu} \lambda^{-|\nu|-n(s-1) / 2} \sigma_{n, \nu}(A)\left(\chi_{n, \nu, \beta}\right)_{\lambda}=e^{-\lambda \beta H} A \Omega
$$

at each fixed $\lambda$, whenever $A$ is a linear combination of Weyl operators, and when evaluated in scalar products with vectors from a dense set. But (7.37) also shows that the left hand side of (7.38) converges in $\mathcal{B}\left(\mathfrak{A}\left(\lambda \mathcal{O}_{r}\right), \mathcal{H}\right)$, and it is therefore strongly continuous for $A$ in norm bounded parts of $\mathfrak{A}\left(\lambda \mathcal{O}_{r}\right)$. Then by Kaplansky's theorem (7.38) holds for any $A \in \mathfrak{A}\left(\lambda \mathcal{O}_{r}\right)$ as an equality in $\mathcal{H}$. Finally, this entails for all $\underline{A} \in \underline{\mathfrak{A}}\left(\mathcal{O}_{r}\right)$ that $\sum_{n, \nu} \underline{\sigma}_{n, \nu}\left(\underline{C}^{<} \underline{A}\right)_{\lambda}\left(\chi_{n, \nu, \beta}\right)_{\lambda}=$ $\underline{\Theta}^{\left(\beta, \mathcal{O}_{r}\right)}(\underline{A})_{\lambda}$ at each fixed $\lambda>0$, i.e. the statement.

We now prove Eq. (7.37). Let $r_{0}>0$ be fixed in the following, and $r<r_{0}$. From Prop.7.8 and Lemma 7.9, we obtain the estimate

$$
\begin{aligned}
\| \underline{\sigma}_{n, \nu}\left\lceil\underline{\mathfrak{A}}\left(\mathcal{O}_{r}\right)\|\| \chi_{n, \nu, \beta} \|\right. & \leq c^{2 n}(6 r / \beta)^{|\nu|+n(s-1) / 2} \\
& =\left(c^{2}(6 r / \beta)^{(s-1) / 2}\right)^{n} \prod_{j=1}^{n}\left((6 r / \beta)^{\nu_{j 0}} \prod_{k=1}^{s}(6 r / \beta)^{\nu_{j k}}\right) .
\end{aligned}
$$

Factorizing the sum over multi-indexes $\nu$ accordingly, we obtain at fixed $n$,

$$
\sum_{\nu} \| \underline{\sigma}_{n, \nu}\left\lceil\underline{\mathfrak{A}}\left(\mathcal{O}_{r}\right)\|\| \underline{\chi}_{n, \nu, \beta} \| \leq\left(2 c^{2} \frac{(6 r / \beta)^{(s-1) / 2}}{(1-6 r / \beta)^{s}}\right)^{n}\right.
$$

where we suppose $6 r / \beta<1$, and where each sum over $\nu_{j 0} \in\{0,1\}$ has been estimated by introducing a factor of 2 . Now if we choose $r / \beta$ small enough, we can certainly achieve that the expression in Eq. (7.40) is summable over $n$ as a geometric series, and hence the series in Eq. (7.37) converges.

This establishes the phase space condition of Def. 6.2 in our context.

Corollary 7.11. The theory of a real scalar free field of mass $m \geq 0$ in $3+1$ or more space-time dimensions fulfills the uniform Haag-Swieca compactness condition.

While our goal was to show that the maps $\underline{\Theta}^{(\beta, \mathcal{O})}$ are uniformly compact, it follows from Eq. (7.37) that they are actually uniformly nuclear at all scales, or by a slightly modified argument, even uniformly $p$-nuclear for any $0<p \leq 1$. So we can generalize the somewhat 
stronger Buchholz-Wichmann condition [BW86] to our context. Several other types of phase space conditions can be derived with similar methods as in Thm. 7.10 as well. Particularly, one can show for $s \geq 3$ that the sum $\sum_{n, \nu} \sigma_{n, \nu} \phi_{n, \nu}$ converges in norm under a cutoff in energy $E$ and restriction to a fixed local algebra $\mathfrak{A}\left(\mathcal{O}_{r}\right)$, with estimates uniform in $E \cdot r$ where this product is small. This implies that Phase Space Condition II of [BDM09], which guarantees a regular behavior of pointlike fields under scaling, is fulfilled for those models.

\section{Conclusions}

In this paper, we have considered short-distance scaling limits in the model independent framework of Buchholz and Verch [BV95]. In order to describe the dilation symmetry that arises in the limit theory, we passed to a generalized class of limit states, which includes states invariant under scaling. The essential results of BV95 carry over to this generalization, including the structure of Poincaré symmetries and geometric modular action.

However, the dilation invariant limit states are not pure. Rather, they can be decomposed into states of the Buchholz-Verch type, which are pure in two or more spatial dimensions. This decomposition gives rise to a direct integral decomposition of the Hilbert space of the limit theory, under which local observables, Poincaré symmetries, and other relevant objects of the theory can be decomposed - except for dilations. The dilation symmetry has a more intricate structure, intertwining the pure components of the limit state.

The situation simplifies if we consider the situation of a "unique limit" in the classification of [BV95]; our condition of a "factorizing limit" turned out to be equivalent modulo technicalities. Under this restriction, the dilation unitaries in the limit are, up to a central part, decomposable operators. The decomposed components do not necessarily fulfill a group relation though, but a somewhat weaker cocycle relation. Only under stronger assumptions ("convergent scaling limits") we were able to show that the dilation symmetry factorizes into a tensor product of unitary group representations.

It is unknown at present which type of models would make the generalized decomposition formulas necessary. In this paper, we have only considered very simple examples, which all turned out to fall into the more restricted class of convergent scaling limits. However, thinking e.g. of infinite tensor products of free fields with increasing masses as suggested in Buc96a, Sec. 5], it may well be that some models violate the condition of uniqueness of the scaling limit, or even exhibit massive particles in the limit theory. In this case, the direct integral decomposition would be needed to obtain a reasonable description of dilation symmetry in the limit, with the symmetry operators intertwining fibers of the direct integral that correspond to different masses.

As a next step in the analysis of dilation symmetries in the limit theory, one would like to investigate further the deviation of the theory at finite scales from the idealized dilation covariant limit theory; so to speak, the next-order term in the approximation $\lambda \rightarrow 0$. This would be interesting e.g. for applications to deep inelastic scattering, which can currently only be treated in formal perturbation theory. It is expected that the dilation symmetry in the limit also contains some information about these next-order terms. Our formalism, however, does at the moment not capture these next-order approximations, and would need to be generalized considerably.

Further, it would be interesting to see whether the dilation symmetry we analyzed can be used to obtain restrictions on the type of interaction in the limit theory, possibly leading 
to criteria for asymptotic freedom. Here we do not refer to restrictions on the form of the Lagrangian, a concept that is not visible in our framework. Rather, we think that dilation symmetries should manifest themselves in the coefficients of the operator product expansion Bos05a, BDM09, BF08, or in the general structure of local observables [BF77].

In this context, it seems worthwhile to investigate simplified low-dimensional interacting models, such as the $1+1$ dimensional massive models with factorizing $\mathrm{S}$ matrix that have recently been rigorously constructed in the algebraic framework [Lec08]. By abstract arguments, these models possess a scaling limit theory in our context. Just as in the Schwinger model [Buc96b, BV98], one expects here that even the limit theory for multiplicative states has a nontrivial center. This may be seen as a peculiarity of the $1+1$ dimensional situation; we have not specifically dealt with this problem in the present paper. But neglecting these aspects, one would expect that the limit theory corresponds to a massless (and dilation covariant) model with factorizing S matrix, although it would probably not have an interpretation in the usual terms of scattering theory. Such models of "massless scattering" have indeed been considered in the physics literature; see [FS94 for a review. Their mathematical status as quantum field theories, however, remains largely unclear at this time. Nevertheless, one should be able to treat them with our methods.

In fact, these examples may give a hint to the restrictions on interaction that the dilation symmetry implies. At least in a certain class of two-particle $S$ matrices - those which tend to 1 at large momenta - one expects that the limit theory is chiral, i.e., factors into a tensor product of two models living on the left and right light ray, respectively. On the other hand, the theories we consider are always local; and for chiral local models, dilation covariance is the essential property that guarantees conformal covariance GLW98. So if those models have a nontrivial scaling limit at all, they underly quite rigid restrictions, since local conformal chiral nets are - at least partially - classifiable in a discrete series KL04. The detailed investigation of these aspects of factorizing $\mathrm{S}$ matrix models is however the subject of ongoing research, and some surprises are likely to turn up.

\section{A Direct integrals of Hilbert spaces}

In our investigation, we make use of the concept of direct integrals of Hilbert spaces, $\mathcal{H}=$ $\int_{\mathcal{Z}} d \nu(z) \mathcal{H}_{z}$, where the integral is defined on some measure space $(\mathcal{Z}, \nu)$. Due to difficult measure-theoretic problems, the standard literature treats these direct integrals only under separability assumptions on the Hilbert spaces involved; see e.g. KR97. These are however not a priori implied in our analysis; and even where we make such assumptions, we need to apply them with care. While we can often reasonably assume the "fiber spaces" $\mathcal{H}_{z}$ to be separable, the measure space $\mathcal{Z}$ will, in our applications, be of a very general nature, and even $L^{2}(\mathcal{Z}, \nu)$ is known to be non-separable in some situations. The concept of direct integrals can be generalized to that case. Since however the literature on that topid 6 is somewhat scattered and not easily accessible, we give here a brief review for the convenience of the reader, restricted to the case that concerns us.

In the following, let $\mathcal{Z}$ be a compact topological space and $\nu$ a finite regular Borel measure

\footnotetext{
${ }^{6}$ In the general case, we largely follow Wils Wil70, however with some changes in notation. Other, somewhat stronger notions of direct integrals exist, e.g. [God51, Ch. III], Seg51; see [Mar69] for a comparison. Under separability assumptions (Definition A.3), all these notions agree, and we are in the case described in Tak79, Ch. IV.8], Dix81, Part II Ch. 1].
} 
on $\mathcal{Z}$. For each $z \in \mathcal{Z}$, we consider a Hilbert space $\mathcal{H}_{z}$ with scalar product $\langle\cdot \mid \cdot\rangle_{z}$ and associated norm $\|\cdot\|_{z}$. Elements $\chi \in \prod_{z \in \mathcal{Z}} \mathcal{H}_{z}$ will be called vector fields and alternatively denoted as maps, $z \mapsto \chi(z)$. Direct integrals of this field of Hilbert spaces $\mathcal{H}_{z}$ over $\mathcal{Z}$ are not unique, but depend on the choice of a fundamental family.

Definition A.1. A fundamental family is a linear subspace $\Gamma \subset \prod_{z \in \mathcal{Z}} \mathcal{H}_{z}$ such that for every $\chi \in \Gamma$, the function $z \mapsto\|\chi(z)\|_{z}^{2}$ is $\nu$-integrable. If the same function is always continuous, we say that $\Gamma$ is a continuous fundamental family.

The continuity aspect will be discussed further below, for the moment we focus on measurability. Each fundamental family $\Gamma$ uniquely extends to a minimal vector space $\bar{\Gamma}$, with $\Gamma \subset \bar{\Gamma} \subset \prod_{z \in \mathcal{Z}} \mathcal{H}_{z}$, which has the following properties. [Wil70, Corollary 2.3]

(i) $z \mapsto\|\chi(z)\|_{z}^{2}$ is $\nu$-integrable for all $\chi \in \bar{\Gamma}$.

(ii) If for $\chi \in \prod_{z} \mathcal{H}_{z}$, there exists $\hat{\chi} \in \bar{\Gamma}$ such that $\chi(z)=\hat{\chi}(z)$ a.e., then $\chi \in \bar{\Gamma}$.

(iii) If $\chi \in \bar{\Gamma}$ and $f \in L^{\infty}(\mathcal{Z}, \nu)$, then $f \chi \in \bar{\Gamma}$, where $(f \chi)(z):=f(z) \chi(z)$.

(iv) $\bar{\Gamma}$ is complete with respect to the seminorm $\|\chi\|=\left(\int_{\mathcal{Z}} d \nu(z)\|\chi(z)\|^{2}\right)^{1 / 2}$.

Such $\bar{\Gamma}$ is called an integrable family. It is obtained from $\Gamma$ by multiplication with $L^{\infty}$ functions and closure in $\|\cdot\|$. The elements of $\bar{\Gamma}$ are called $\Gamma$-measurable functions; they are in fact analogues of square-integrable functions, and the usual measure theoretic results hold for them: Egoroff's theorem; the dominated convergence theorem; any norm-convergent sequence $\left(\chi_{n}\right)$ in $\bar{\Gamma}$ has a subsequence on which $\chi_{n}(z)$ converges pointwise a.e.; and if $\left(\chi_{n}\right)$ is a sequence in $\bar{\Gamma}$ that converges pointwise a.e., the limit function $\chi$ is in $\bar{\Gamma}$. (Cf. Propositions 1.3 and 1.5 of [Mar69.) After dividing out vectors of zero norm (which we do not denote explicitly), $\bar{\Gamma}$ becomes a Hilbert space, which we call the direct integral of the $\mathcal{H}_{z}$ with respect to $\Gamma$, and denote it as

$$
\mathcal{H}=\int_{\mathcal{Z}}^{\Gamma} d \nu(z) \mathcal{H}_{z}, \quad \text { with scalar product }(\chi \mid \hat{\chi})=\int_{\mathcal{Z}} d \nu(z)\langle\chi(z) \mid \hat{\chi}(z)\rangle_{z} .
$$

Correspondingly, the elements $\chi \in \mathcal{H}$ are denoted as $\chi=\int_{\mathcal{Z}}^{\Gamma} \chi(z) d \nu(z)$.

We also consider bounded operators between such direct integral spaces. Let $\mathcal{H}_{z}, \hat{\mathcal{H}}_{z}$ be two fields of Hilbert spaces over the same measure space $\mathcal{Z}$, and let $\Gamma, \hat{\Gamma}$ be associated fundamental families. We call $B \in \prod_{z \in \mathcal{Z}} \mathcal{B}\left(\mathcal{H}_{z}, \hat{\mathcal{H}}_{z}\right)$ a measurable field of operators if $\operatorname{ess}_{\sup _{z}}\|B(z)\|<\infty$, and if for every $\chi \in \bar{\Gamma}$, the vector field $z \mapsto B(z) \chi(z)$ is $\hat{\Gamma}$-measurable, i.e. an element of $\overline{\hat{\Gamma}}$. In fact it suffices to check the measurability condition on the fundamental family $\Gamma$.

Lemma A.2. Let $B \in \prod_{z \in \mathcal{Z}} \mathcal{B}\left(\mathcal{H}_{z}, \hat{\mathcal{H}}_{z}\right)$ such that $\operatorname{ess}_{\sup }\|B(z)\|<\infty$, and such that $z \mapsto$ $B(z) \chi(z)$ is $\hat{\Gamma}$-measurable for every $\chi \in \Gamma$. Then $B$ is a measurable field of operators.

Proof. Evidently, $z \mapsto B(z) \chi(z)$ is also $\hat{\Gamma}$-measurable if $\chi$ is taken from $L^{\infty}(\mathcal{Z}, \nu) \cdot \Gamma$ or from its linear span. This span is however dense in $\mathcal{H}$. So let $\chi \in \mathcal{H}$. There exists a sequence $\chi_{n} \in \operatorname{span} L^{\infty}(\mathcal{Z}, \nu) \cdot \Gamma$ such that $\chi_{n} \rightarrow \chi$ in norm; by the remarks after Def. A.1, we can assume that $\chi_{n}(z) \rightarrow \chi(z)$ a. e. But then $B(z) \chi_{n}(z) \rightarrow B(z) \chi(z)$ a. e., due to continuity of each $B(z)$. So $B(z) \chi(z)$ is a pointwise a. e. limit of functions in $\hat{\Gamma}$. This implies $(z \mapsto B(z) \chi(z)) \in \overline{\hat{\Gamma}}$, which was to be shown. 
A measurable field of operators $B$ now defines a bounded linear operator $\mathcal{H} \rightarrow \hat{\mathcal{H}}$ which we denote as $B=\int_{\mathcal{Z}}^{\Gamma, \hat{\Gamma}} d \nu(z) B(z)$. Operators in $\mathcal{B}(\mathcal{H}, \hat{\mathcal{H}})$ of this form are called decomposable. Their decomposition need not be unique however, not even a. e. If here $\Gamma=\hat{\Gamma}$, and if $B(z)=f(z) \mathbf{1}_{\mathcal{H}_{z}}$ with a function $f \in L^{\infty}(Z, \nu)$, then $B$ is called a diagonalizable operator. We sometimes write this multiplication operator as $M_{f}=\int_{\mathcal{Z}}^{\Gamma} d \nu(z) f(z) \mathbf{1}$.

Let $\mathfrak{A}$ be a $\mathrm{C}^{*}$ algebra, and let for each $z \in \mathcal{Z}$ a representation $\pi_{z}$ of $\mathfrak{A}$ on $\mathcal{H}_{z}$ be given, such that $z \mapsto \pi_{z}(A)$ is a measurable field of operators for any $A \in \mathfrak{A}$. Then, $\pi(A)=\int_{\mathcal{Z}}^{\Gamma} d \nu(z) \pi_{z}(A)$ defines a new representation $\pi$ of $A$ on $\mathcal{H}$, which we formally denote as $\pi=\int_{\mathcal{Z}}^{\Gamma} d \nu(z) \pi_{z}$.

In many cases, obtaining useful results regarding decomposable operators requires additional separability assumptions. The following property will usually be general enough for us.

Definition A.3. $A$ fundamental sequence in $\mathcal{H}=\int_{\mathcal{Z}}^{\Gamma} d \nu(z) \mathcal{H}_{z}$ is a sequence $\left(\chi_{j}\right)_{j \in \mathbb{N}}$ in $\bar{\Gamma}$ such that for every $z \in \mathcal{Z}$, the set $\left\{\chi_{j}(z) \mid j \in \mathbb{N}\right\}$ is total in $\mathcal{H}_{z}$. If such a fundamental sequence exists for $\mathcal{H}$, we say that $\mathcal{H}$ is uniformly separable.

This more restrictive situation agrees with the setting of [Tak79, Dix81]; cf. [Mar69, Prop. 1.13]. Note that this property implies that the fiber spaces $\mathcal{H}_{z}$ are separable, but the integral space $\mathcal{H}$ does not need to be separable if $\mathcal{Z}$ is sufficiently general. Under the above separability assumption, additional desirable properties of decomposable operators hold true.

Theorem A.4. Let $\mathcal{H}=\int_{\mathcal{Z}}^{\Gamma} d \nu(z) \mathcal{H}_{z}$ and $\hat{\mathcal{H}}=\int_{\mathcal{Z}}^{\hat{\Gamma}} d \nu(z) \hat{\mathcal{H}}_{z}$ both be uniformly separable. Then, for each decomposable operator $B=\int_{\mathcal{Z}}^{\Gamma, \hat{\Gamma}} d \nu(z) B(z)$, also $B^{*}$ is decomposable, with $B^{*}=\int_{\mathcal{Z}}^{\hat{\Gamma}, \Gamma} d \nu(z) B(z)^{*}$. One has $\|B\|=\left\|B^{*}\right\|=\operatorname{ess}_{\sup _{z}}\|B(z)\|$. Decompositions of operators are unique in the following sense: If $\int_{\mathcal{Z}}^{\Gamma, \hat{\Gamma}} d \nu(z) B(z)=\int_{\mathcal{Z}}^{\Gamma, \hat{\Gamma}} d \nu(z) \hat{B}(z)$, then $B(z)=\hat{B}(z)$ for almost every $z$.

For the proof methods, see e.g. God51, Ch. III Sec. 13]. Note that the theorem is false if the separability assumption is dropped; see Example 7.6 and Remark 7.11 of [Tak79, Ch. IV]. We also obtain an important characterization of decomposable operators.

Theorem A.5. Let $\mathcal{H}, \hat{\mathcal{H}}$ be uniformly separable. An operator $B \in \mathcal{B}(\mathcal{H}, \hat{\mathcal{H}})$ is decomposable if and only if it commutes with all diagonalizable operators; i.e. $M_{f} B=B \hat{M}_{f}$ for all $f \in$ $L^{\infty}(Z, \nu)$.

A proof can be found in [Dix81, Ch. II $\S 2$ Sec. 5 Thm. 1]. In particular, if $\mathcal{H}=\hat{\mathcal{H}}$, we know that both the decomposable operators and the diagonalizable operators form $\mathrm{W}^{*}$ algebras, which are their mutual commutants. Note that the "if" part of the theorem is known to be false for sufficiently general direct integrals, violating the separability assumption [Sch90].

We now discuss the case of a continuous fundamental family $\Gamma$; cf. God51, Ch. III Sec. 2]. In this case, we can consider the space of $\Gamma$-continuous functions, denoted $\mathcal{C}(\Gamma)$, and defined as the closed span of $\mathcal{C}(\mathcal{Z}) \cdot \Gamma$ in the supremum norm, $\|\chi\|_{\infty}=\sup _{z \in \mathcal{Z}}\|\chi(z)\|_{z}$. With this norm, $\mathcal{C}(\Gamma)$ is a Banach space, in fact a Banach module over the commutative $\mathrm{C}^{*}$ algebra $\mathcal{C}(\mathcal{Z})$. We have $\mathcal{C}(\Gamma) \subset \bar{\Gamma}$ in a natural way, and this inclusion is dense, but it is important to note that different norms are used in these two spaces.

A simple but particularly important example for direct integrals arises as follows Tak79, Ch. IV.7]. Let $\mathcal{H}_{\mathrm{u}}$ be a fixed Hilbert space, and $\mathcal{Z}$ a measure space as above. For each $z \in \mathcal{Z}$, 
set $\mathcal{H}_{z}=\mathcal{H}_{\mathrm{u}}$. Then the set $\Gamma$ of constant functions $\mathcal{Z} \rightarrow \mathcal{H}_{\mathrm{u}}$ is a continuous fundamental family; and the associated integrable family $\bar{\Gamma}$ is precisely the space of all square-integrable, Lusin-measurable functions $\chi: \mathcal{Z} \rightarrow \mathcal{H}_{\mathrm{u}}$. We denote the corresponding direct integral space as $L^{2}\left(\mathcal{Z}, \nu, \mathcal{H}_{\mathrm{u}}\right)=\int_{\mathcal{Z}}^{\oplus} d \nu(z) \mathcal{H}_{\mathrm{u}}$ (with reference to the "canonical" fundamental family). This space is isomorphic to $L^{2}(\mathcal{Z}, \nu) \otimes \mathcal{H}_{\mathrm{u}}$; the canonical isomorphism, which we do not denote explicitly, maps $f \otimes \chi$ to the function $z \mapsto f(z) \chi$. In this way, the algebra of diagonal operators is identified with $L^{\infty}(\mathcal{Z}, \nu) \otimes \mathbf{1}$.

If here $\mathcal{H}_{\mathrm{u}}$ is separable, then $L^{2}\left(\mathcal{Z}, \nu, \mathcal{H}_{\mathrm{u}}\right)$ is clearly uniformly separable. In this case, a simple criterion identifies the elements of the integral space: A function $\chi: \mathcal{Z} \rightarrow \mathcal{H}_{\mathrm{u}}$ is Lusin measurable if and only if it is weakly measurable, i.e. if $z \mapsto\langle\chi(z) \mid \eta\rangle$ is measurable for any fixed $\eta \in \mathcal{H}_{\mathrm{u}}$. Also, the algebra of decomposable operators is isomorphic to $L^{\infty}(\mathcal{Z}, \nu) \bar{\otimes} \mathcal{B}\left(\mathcal{H}_{\mathrm{u}}\right)$.

\section{Acknowledgements}

The authors are obliged to Laszlo Zsido and Michael Müger for helpful discussions. They also profited from financial support by the Erwin Schrödinger Institute, Vienna, and from the friendly atmosphere there. HB further wishes to thank the II. Institut für Theoretische Physik, Hamburg, for their hospitality.

\section{References}

[Ara64] Huzihiro Araki. On the algebra of all local observables. Prog. Theoret. Phys., 32:844-854, 1964 .

[BDF87] Detlev Buchholz, Claudio D'Antoni, and Klaus Fredenhagen. The universal structure of local algebras. Commun. Math. Phys., 111:123-135, 1987.

[BDM09] Henning Bostelmann, Claudio D'Antoni, and Gerardo Morsella. Scaling algebras and pointlike fields. A nonperturbative approach to renormalization. Commun. Math. Phys., 285:763-798, 2009.

[BF77] Detlev Buchholz and Klaus Fredenhagen. Dilations and interactions. J. Math. Phys., 18:1107-1111, 1977.

[BF08] Henning Bostelmann and Christopher J. Fewster. Quantum inequalities from operator product expansions. arXiv:0812.4760, 2008.

[Bos00] Henning Bostelmann. Lokale Algebren und Operatorprodukte am Punkt. Thesis, Universität Göttingen, 2000. Available online at http://webdoc.sub.gwdg.de/diss/2000/bostelmann/.

[Bos05a] Henning Bostelmann. Operator product expansions as a consequence of phase space properties. J. Math. Phys., 46:082304, 2005.

[Bos05b] Henning Bostelmann. Phase space properties and the short distance structure in quantum field theory. J. Math. Phys., 46:052301, 2005.

[BP90] Detlev Buchholz and Martin Porrmann. How small is the phase space in quantum field theory? Ann. Inst. H. Poincaré, 52:237-257, 1990. 
[BR79] Ola Bratteli and Derek W. Robinson. Operator Algebras and Quantum Statistical Mechanics, volume I. Springer, New York, 1979.

[BR81] Ola Bratteli and Derek W. Robinson. Operator Algebras and Quantum Statistical Mechanics, volume II. Springer, New York, 1981.

[Buc96a] Detlev Buchholz. Phase space properties of local observables and structure of scaling limits. Ann. Inst. H. Poincaré, 64:433-459, 1996.

[Buc96b] Detlev Buchholz. Quarks, gluons, colour: facts or fiction? Nucl. Phys. B, 469:333353, 1996.

[BV95] Detlev Buchholz and Rainer Verch. Scaling algebras and renormalization group in algebraic quantum field theory. Rev. Math. Phys., 7:1195-1239, 1995.

[BV98] Detlev Buchholz and Rainer Verch. Scaling algebras and renormalization group in algebraic quantum field theory. II. Instructive examples. Rev. Math. Phys., 10:775800, 1998.

[BW86] Detlev Buchholz and Eyvind H. Wichmann. Causal independence and the energylevel density of states in local quantum field theory. Commun. Math. Phys., 106:321344, 1986.

[Dix81] Jacques Dixmier. Von Neumann Algebras. North-Holland, Amsterdam, 1981.

[DM06] Claudio D'Antoni and Gerardo Morsella. Scaling algebras and superselection sectors: Study of a class of models. Rev. Math. Phys., 18:565-594, 2006.

[DMV04] Claudio D'Antoni, Gerardo Morsella, and Rainer Verch. Scaling algebras for charged fields and short-distance analysis for localizable and topological charges. Annales Henri Poincare, 5:809-870, 2004.

[DS85] Wulf Driessler and Stephen J. Summers. Central decomposition of Poincaréinvariant nets of local field algebras and absence of spontaneous breaking of the Lorentz group. Ann. Inst. H. Poincaré Phys. Theor., 43:147-166, 1985.

[EF74] J. P. Eckmann and J. Fröhlich. Unitary equivalence of local algebras in the quasifree representation. Ann. Inst. H. Poincaré Sect. A (N.S.), 20:201-209, 1974.

[FS94] P. Fendley and H. Saleur. Massless integrable quantum field theories and massless scattering in 1+1 dimensions. In E. Gava, A. Masiero, K. S. Nariain, S. RandjbarDaemi, and Q. Shafi, editors, Proceedings of the 1993 Summer School on High Energy Physics and Cosmology, volume 10 of ICTP Series in Theoretical Physics. World Scientific, 1994.

[GLW98] D. Guido, R. Longo, and H. W. Wiesbrock. Extensions of conformal nets and superselection structures. Commun. Math. Phys., 192:217-244, 1998.

[God51] R. Godement. Sur la théorie des représentations unitaires. Ann. of Math., 53:68$124,1951$.

[Haa96] Rudolf Haag. Local Quantum Physics. Springer, Berlin, 2nd edition, 1996. 
[HS65] Rudolf Haag and J. A. Swieca. When does a quantum field theory describe particles? Commun. Math. Phys., 1:308-320, 1965.

[KL04] Yasuyuki Kawahigashi and Roberto Longo. Classification of local conformal nets. Case $c<1$. Ann. of Math., 160(2):493-522, 2004.

[KR97] Richard V. Kadison and John R. Ringrose. Fundamentals of the Theory of Operator Algebras, volume II: Advanced Theory. Academic Press, Orlando, 1997.

[Lec08] Gandalf Lechner. Construction of quantum field theories with factorizing $S$ matrices. Commun. Math. Phys., 277(3):821-860, 2008.

[Mar69] Odile Maréchal. Champs mesurables d'espaces hilbertiens. Bull. Sci. Math., 93:113143, 1969.

[Mit66] Theodore Mitchell. Fixed points and multiplicative left invariant means. Trans. Amer. Math. Soc., 122:195-202, 1966.

[Sch90] Reinhard Schaflitzel. The algebra of decomposable operators in direct integrals of not necessarily separable Hilbert spaces. Proc. Amer. Math. Soc., 110:983-987, 1990 .

[Seg51] I. Segal. Decompositions of Operator Algebras I, II, volume 9 of Mem. Amer. Math. Soc. American Mathematical Society, Providence, R.I., 1951.

[Tak79] Masamichi Takesaki. Theory of Operator Algebras I. Springer, Berlin, 1979.

[Tak02] Masamichi Takesaki. Theory of Operator Algebras II. Springer, Berlin, 2002.

[Wil70] Wilbert Wils. Direct integrals of Hilbert spaces I. Math. Scand., 26:73-88, 1970. 